\title{
THE NONHOLONOMIC RIEMANN AND WEYL TENSORS FOR FLAG MANIFOLDS
}

\author{
PAVEL GROZMAN ${ }^{1}$, DIMITRY LEITES ${ }^{2}$
}

\begin{abstract}
On any manifold, any non-degenerate symmetric 2-form (metric) and any skewsymmetric (differential) form $\omega$ can be reduced to a canonical form at any point, but not in any neighborhood: the respective obstructions being the Riemannian tensor and $d \omega$. The obstructions to flatness (to reducibility to a canonical form) are well-known for any $G$-structure, not only for Riemannian or symplectic structures.

For the manifold with a nonholonomic structure (nonintegrable distribution), the general notions of flatness and obstructions to it, though of huge interest (e.g., in supergravity) were not known until recently, though particular cases were known for more than a century (e.g., any contact structure is "flat": it can always be reduced, locally, to a canonical form).

We give a general definition of the nonholonomic analogs of the Riemann and Weyl tensors. With the help of Premet's theorems and a package SuperLie we calculate these tensors for the particular case of flag varieties associated with each maximal (and several other) parabolic subalgebra of each simple Lie algebra. We also compute obstructions to flatness of the $G(2)$-structure and its nonholonomic super counterpart.
\end{abstract}

To Antony Joseph

\section{INTRODUCTION}

H. Hertz $[\mathrm{H}]$ coined the term nonholonomic during his attempts to geometrically describe motions in such a way as to exorcize the concept of "force". A manifold (phase space) is said to be nonholonomic if endowed with a nonintegrable distribution (a subbundle of the tangent bundle). A simplest example of a nonholonomic dynamical system is given by a body rolling without gliding over another body. Among various images that spring to mind, the simplest is a ball on a rough plane ([Poi] $)$ or a bike on asphalt. At the tangency point of the wheel with asphalt, the velocity of the wheel is zero. (This is a linear constraint. We will not consider here more general non-linear constraints.) A famous theorem of Frobenius gives criteria of local integrability of the distribution: its sections should form a Lie algebra.

For a historical review of nonholonomic systems, see VG2 and a very interesting paper by Vershik [V] with first rigorous mathematical formulations of nonholonomic geometry and indications to applications to various, partly unexpected at that time, areas (like optimal control or macro-economics, where nonlinear constraints are also natural, cf. [AS], Bl], $[\mathrm{S}]$ ); recent book by Kozlov [Koz is extremely instructive. In [V], Vershik summarizes about 100 years of studies of nonholonomic geometry (Hertz, Carathéodory, Vrănceanu, Wagner, Schouten, Faddeev, Griffiths, Godbillon; now MathSciNet returns thousands entries for "nonholonomic" and its synonyms (anholonomic, "sub-Riemannian", "autoparallel") and

1991 Mathematics Subject Classification. 17A70 (Primary) 17B35 (Secondary).

Key words and phrases. Lie algebra cohomology, Cartan prolongation, Riemann tensor, nonholonomic manifold, flag manifolds, $G_{2}$-structure.

DL thankfully acknowledges help of A. Premet, I. Shchepochkina and J. Bernstein and financial support of IHES, Försäkringskassan and MPIMiS. 
particular cases leading to nonholonomic constraints ("Finsler", "cat's problem"). There seems to be "more", actually, nonholonomic dynamical systems than holonomic ones.

A relatively new theory, "supergravity" (the theory embodying Einstein's dream of a Unified Field Theory), also deals exclusively with nonholonomic structures, albeit on supermanifolds.)

At the end of [V] Vershik summarized futile attempts of the researchers to define an analog of the Riemann tensor for the general nonholonomic manifold in a conjecture that "though known in some cases, it is probably impossible to define such a general analog".

However, in 1989, during his stay at IAS, DL gave such a general definition and lectured on it at various schools and conferences (ICTP, Euler Math. Inst., JINR, etc.), see [L], LP]; later we applied it to supergravity [GL1].

Main results of this paper are (1) elucidation of this general definition of the nonholonomic counterparts of the Riemann tensor and its conformal, Weyl, analog; (2) Premet's theorems that facilitate computation of these tensors in some cases (for flag varieties $G / P$, where $G$ is a simple Lie group and $P$ is its parabolic subalgebra); (3) computation of these tensors in some of these cases.

Tanaka [T] tackled the same, actually, problem for totally different reasons (in [T] and even in more recent $[\mathrm{Y}]$ and $[\mathrm{YY}]$ even the word "nonholonomic" is never used). Tanaka's results (especially their lucid exposition in Yamaguchi's paper [Y]) are easier to understand than the first attempts (by Schouten, Wagner, see [DG]) because, after some experiments, he used the hieroglyphics of Lie algebra cohomology which are much more graphic than coordinate tensor notations. Tanaka's tensor coincides with the one we suggest.

We illustrate the main definitions by computing the nonholonomic analogs of the Riemann and Weyl tensor in several particular cases - the simplest analogs of "classical domains". In doing so we rely on Premet's theorems and a Mathematica-based package SuperLie.

Computations of nonholonomic analogs of Riemann tensor are rather difficult technically and the rare examples of works with actually computed results are C1]- C5], GIOS, [HH], $\mathrm{Y}, \mathrm{YY}, \mathrm{EKMR}, \mathrm{Ta}$ and refs. therein. In non-super setting, they used Tanaka's definition of nonholonomic Riemann tensor, identical to ours, but lack Premet's theorems and SuperLie and so could not compute as much as anybody is able to now with their help. ${ }^{1}$

0.1. General description of classical tensors and our examples. In mid-1970s, Gindikin formulated a problem of local characterization of compact Hermitian symmetric domains $X=S / P$, where $S$ is a simple Lie group and $P$ its parabolic subgroup. Goncharov solved this problem [Go] having considered the fields of certain quadratic cones and having computed the structure functions (obstructions to flatness) of the corresponding $G$-structures, where $G$ is the Levi (reductive) part of $P$.

0.1.1. Examples. Let the ground field be $\mathbb{C}$.

1) For $S=O(n+2)$ and $G=C O(n)=O(n) \times \mathbb{C}^{\times}$, the structure functions were known; they constitute the Weyl tensor — the conformally invariant part of the Riemann curvature tensor.

2) For $S=S L(n+m)$ and $G=S(G L(n) \times G L(m))$, the structure functions are obstructions to integrability of multidimensional analogs of Penrose's $\alpha$ - and $\beta$-planes on the Grassmannian $G r_{n}^{n+m}$ (Penrose considered $G r_{2}^{4}$ ).

\footnotetext{
${ }^{1}$ In 2000, S. Vacaru informed us of his and mathematician's from Vrănceanu's school definitions partly summarized in $\mathrm{Va6}$ and refs. therein. It is not easy to see through the forest of non-invariant cumbrous tensor expressions that a number of components is lacking in Va6] as well as in DG], as compared with Tanaka's or our definitions.
} 
Not any simple complex Lie group $S$ and its subgroup $P$ can form a classical domain: $S$ is any but $G(2)^{2}, F(4)$ and $E(8)$ and $P=P_{i}$ is a maximal parabolic subgroup generated by all Chevalley generators of $S$, but one ( $i$ th), say, negative. The group $P$, or which is the same, the $i$ th Chevalley generator of $S$ (in what follows referred to as selected) can not be arbitrary, either. To describe the admissible $P$ 's, let us label the nodes of the Dynkin graph of $S$ with the coefficients of the maximal root expressed in terms of simple roots. The selected generator may only correspond to the vertex with label 1 on the Dynkin graph.

It is natural to consider the following problem:

For any simple Lie group $S$, fix an arbitrary $\mathbb{Z}$-grading of its Lie algebra $\mathfrak{s}=\operatorname{Lie}(S)$.

For any subgroup $P \subset S$, generated by nonnegative elements of $\mathfrak{s}$, what are the analogs of the Goncharov conformal structure and the corresponding analogs of Riemann and projective structures, which of these structures are flat,
and what are the obstructions to their flatness?

0.1.2. Remark. The adjective "arbitrary" (Z्Z-grading of $\mathfrak{s})$ in $(0.1)$ appeared thanks to J. Bernstein who reminded us that parabolic subgroups are a particular case of such gradings. All $\mathbb{Z}$-gradings are obtained by setting $\operatorname{deg} X_{i}^{ \pm}= \pm k_{i}$, where $k_{i} \in \mathbb{Z}$, for the Chevalley generators $X_{i}^{ \pm}$and parabolic subgroups appear if $k_{i} \geq 0$ for all $i$. Recently Kostant $\mathrm{K}$ ] considered an analog of the Borel-Weil-Bott (BWB) theorem - one of our main tools for the non-parabolic case, but the answer is not yet as algebraic as we need, so having answered questions (0.1) in full generality we calculate the nonholonomic invariants for parabolic subgroups only.

Modern descriptions of structure functions is usually given in terms of the Spencer cohomology, cf. [St] (we will recall all definitions needed in (0.1) and (0.2) in due course). Goncharov expressed the structure functions as tensors taking values in the vector bundle over $X=G / P$, whose fibers at every point $x \in X$ are isomorphic to each other and to

$$
\begin{aligned}
& H^{2}\left(\mathfrak{g}_{-1} ;\left(\mathfrak{g}_{-1}, \mathfrak{g}_{0}\right)_{*}\right), \quad \text { where } \mathfrak{g}_{0}=\operatorname{Lie}(G), \quad \mathfrak{g}_{-1}=T_{x} X, \\
& \text { and where }\left(\mathfrak{g}_{-1}, \mathfrak{g}_{0}\right)_{*}=\underset{i \geq-1}{\oplus} \mathfrak{g}_{i} \text { is the Cartan prolong of }\left(\mathfrak{g}_{-1}, \mathfrak{g}_{0}\right) .
\end{aligned}
$$

The conventional representation of the structure functions as bigraded Spencer cohomology $H^{k, 2}$ can be recovered any time as the homogeneous degree $k$ component of $H^{2}\left(\mathfrak{g}_{-1} ;\left(\mathfrak{g}_{-1}, \mathfrak{g}_{0}\right)_{*}\right)$ corresponding to the $\mathbb{Z}$-grading of $\left(\mathfrak{g}_{-1}, \mathfrak{g}_{0}\right)_{*}$.

At about the same time Goncharov got his result, physicists trying to write down various supergravity equations (for standard or "exotic" $N$-SUGRAs, see [WB, MaG], GIOS], $[\mathrm{HH}]$ ) bumped into the same problem (0.1) with the supergroup $S=S L(4 \mid N)$ for $N \leq 8$ and $P$ generated by all the (analogs of the) Chevalley generators of $G$ but two. The corresponding coset superspace $X$ is a flag supervariety and the difficulties with SUGRAs spoken about, e.g., in WB] ("we do not know how to define the analog of the Riemann tensor for $N>2$ ", in other words: we do not know what might stand in the left-hand sides of the SUGRA equations), were caused not by a super nature of $X$ but by its nonholonomic nature.

Shchepochkina introduced nonholonomic generalizations $\left(\mathfrak{g}_{-}, \mathfrak{g}_{0}\right)_{*}$ of Cartan prolongation $\left(\mathfrak{g}_{-1}, \mathfrak{g}_{0}\right)_{*}$ for needs of our classification of simple infinite dimensional Lie superalgebras of vector fields ([LSh $)$. She introduced them (together with several new types of prolongation, e.g., partial prolongation) in [Sh1], Sh2], Sh14]. These generalizations are precisely what is

\footnotetext{
${ }^{2}$ We denote the exceptional groups and their Lie algebras in the same way as the serial ones, like $S L(n)$; we thus avoid confusing $\mathfrak{g}(2)$ with the second component $\mathfrak{g}_{2}$ of a $\mathbb{Z}$-grading of a Lie algebra $\mathfrak{g}$.
} 
needed to define the nonholonomic analog of the Weyl and Riemann tensors in the general case.

Observe that our nonholonomic invariants, though natural analogs of the curvature and torsion tensors, do not coincide on nonholonomic manifolds with the classical ones and bearing the same name. Indeed, on any nonholonomic manifold, there is, by definition, a nonzero classical torsion (the Frobenius form that to a pair of sections of the distribution assigns their bracket) while, for example, every contact manifold is flat in our sense. To avoid confusion, we should always add adjective "nonholonomic" for the invariants introduced below. Since this is too long, we will briefly say $N$-curvature tensor and specify its degree (=the order of the structure function) if needed; to require vanishing of the torsion is analogous of imposing Wess-Zumino constraints WB].

The main thing is to answer the questions (0.1). Having done this (having given appropriate definitions in the general case of manifolds with nonholonomic structure) we explicitly compute the analogs of (0.2) — the space of nonholonomic structure functions - possible values of the nonholonomic versions of the Weyl and Riemann tensors. We do so for the simplest nonholonomic flag manifolds of the form $S / P$ with one selected Chevalley generator. In most of our cases $\left(\mathfrak{g}_{-}, \mathfrak{g}_{0}\right)_{*}=\mathfrak{s}$, the Lie algebra of $S$, and therefore we can apply the Borel-Weil-Bott (BWB) theorem (reproduced below; for a nice review, see Wo $)$. If $\left(\mathfrak{g}_{-}, \mathfrak{g}_{0}\right)_{*}$ strictly contains $\mathfrak{s}$, we consider the values of cocycles in $\mathfrak{s}$ as well as in $\left(\mathfrak{g}_{-}, \mathfrak{g}_{0}\right)_{*}$.

We cite Premet's theorems that show how to compute the N-Weyl and N-Riemann tensors and use the theorems to get an explicit answer.

The implicit form of the answer in Go hides phenomena manifest if the answer is explicit, as in [LPS, where, thanks to an explicit form of the answer we suggested some analogs of Einstein equations (EE) for certain Grassmannians. For the cases we consider here, a phenomenon similar to that observed in [LPS] is manifest, e.g., for the nodes at the base of the forks in $\mathfrak{e}(6)$ and $\mathfrak{o}(8)$. We intend to consider the related analogs of EE elsewhere.

We illustrate usefulness of computer-aided study by using SuperLie to compute the structure functions for the $G(2)$-structure, so popular lately, cf. [AW, B, FG]. SuperLie already proved useful in many instances (see GL), and is indispensable for Lie superalgebras: for practically all of them, there exists nothing as neat as the BWB theorem ([PS]). We also apply SuperLie to compute the structure functions for a super version of the $G(2)$-structure on the projective superspace $\mathbb{C} P^{1,7}$ with a nonholonomic distribution.

\section{§1. Structure Functions of $G$-Structures}

Let $M^{n}$ be a manifold over a field $\mathbb{K}$. Let $F M$ be the frame bundle over $M$, i.e., the principal $G L(n)$-bundle. Let $G \subset G L(n)$ be a Lie group. A $G$-structure on $M$ is a reduction of the principal $G L(n)$-bundle to the principal $G$-bundle. Another formulation is more understandable: a $G$-structure is a selection of transition functions from one coordinate patch to another so that they belong to $G$ for every intersecting pair of patches.

Thus, in the definition of $G$-structure the following characters participate: $M^{n}$ and two vector bundles over it: $T M$ and $F M$ and the two groups $G \subset G L(n)$ both acting in each fiber of each bundle.

The simplest $G$-structure is the flat $G$-structure defined as follows. For a model manifold with the flat $G$-structure we take $V=\mathbb{C}^{n}$ with a fixed frame. The key moment is identification of the tangent spaces $T_{v} V$ at distinct points $v$. This is performed by means of parallel translations along $v$. This means that we consider $V$ as a commutative Lie group and identify the tangent spaces to it at various points with its Lie algebra, $\mathfrak{v}$. Thanks to 
commutativity:

$\mathfrak{v}$ can be naturally identified with $V$ itself;

it does not matter whether we use left or right translations.

In this way, we get a fixed frame in every $T_{v} V$. The flat $G$-structure is the bundle over $V$ whose fiber over $v \in V$ consists of all frames obtained from the fixed one under the $G$-action. In textbooks on differential geometry (e.g., in $[\mathrm{St}]$ ), the obstructions to identification of the $k$ th infinitesimal neighborhood of a point $m \in M$ on a manifold $M$ with $G$-structure with the $k$ th infinitesimal neighborhood of a point of the manifold $V$ with the above flat $G$-structure are called structure functions of order $k$.

To precisely describe the structure functions, set

$$
\mathfrak{g}_{-1}=T_{m} M, \quad \mathfrak{g}_{0}=\mathfrak{g}=\operatorname{Lie}(G) .
$$

Recall that, for any (finite dimensional) vector space $V$, we have

$$
\operatorname{Hom}(V, \operatorname{Hom}(V, \ldots, \operatorname{Hom}(V, V) \ldots)) \simeq L^{i}(V, V, \ldots, V ; V),
$$

where $L^{i}$ is the space of $i$-linear maps and we have $(i+1)$-many $V$ 's on both sides. Now, we recursively define, for any $i>0$ :

$$
\begin{aligned}
& \mathfrak{g}_{i}=\left\{X \in \operatorname{Hom}\left(\mathfrak{g}_{-1}, \mathfrak{g}_{i-1}\right) \mid X\left(v_{1}\right)\left(v_{2}, v_{3}, \ldots, v_{i+1}\right)=X\left(v_{2}\right)\left(v_{1}, v_{3}, \ldots, v_{i+1}\right)\right. \\
& \text { where } \left.v_{1}, \ldots, v_{i+1} \in \mathfrak{g}_{-1}\right\} .
\end{aligned}
$$

Let the $\mathfrak{g}_{0}$-module $\mathfrak{g}_{-1}$ be faithful. Then, clearly,

$$
\left.\left(\mathfrak{g}_{-1}, \mathfrak{g}_{0}\right)_{*} \subset \mathfrak{v e c t}(n)=\mathfrak{d e r} \mathbb{C}\left[x_{1}, \ldots, x_{n}\right]\right] \text {, where } n=\operatorname{dim} \mathfrak{g}_{-1} .
$$

It is subject to an easy verification that the Lie algebra structure on $\mathfrak{v e c t}(n)$ induces same on $\left(\mathfrak{g}_{-1}, \mathfrak{g}_{0}\right)_{*}$. (It is also easy to see that even if $\mathfrak{g}_{-1}$ is not a faithful $\mathfrak{g}_{0}$-module $\left(\mathfrak{g}_{-1}, \mathfrak{g}_{0}\right)_{*}$ is a Lie algebra, but can not be embedded into $\mathfrak{v e c t}\left(\mathfrak{g}_{-1}^{*}\right)$.) The Lie algebra $\left(\mathfrak{g}_{-1}, \mathfrak{g}_{0}\right)_{*}$ will be called the Cartan's prolong (the result of Cartan's prolongation) of the pair $\left(\mathfrak{g}_{-1}, \mathfrak{g}_{0}\right)$. The Cartan prolong is the Lie algebra of symmetries of the $G$-structure in the space $T_{m} M$.

Let $E^{i}$ be the operator of the $i$ th exterior power, $V^{*}$ the dual of $V$. Set

$$
C_{\left(\mathfrak{g}_{-1}, \mathfrak{g}_{0}\right)_{*}}^{k, s}=\mathfrak{g}_{k-s} \otimes E^{s}\left(\mathfrak{g}_{-1}^{*}\right) .
$$

The differential $\partial_{s}: C_{\left(\mathfrak{g}_{-1}, \mathfrak{g}_{0}\right)_{*}}^{k, s} \longrightarrow C_{\left(\mathfrak{g}_{-1}, \mathfrak{g}_{0}\right)_{*}}^{k, s+1}$ is given by (as usual, the slot with the hatted variable is to be ignored):

$$
\left(\partial_{s} f\right)\left(v_{1}, \ldots, v_{s+1}\right)=\sum_{i}(-1)^{i}\left[f\left(v_{1}, \ldots, \widehat{v_{s+1-i}}, \ldots, v_{s+1}\right), v_{s+1-i}\right]
$$

for any $v_{1}, \ldots, v_{s+1} \in \mathfrak{g}_{-1}$. As expected, $\partial_{s} \partial_{s+1}=0$. The homology of this bicomplex is called Spencer cohomology of the pair $\left(\mathfrak{g}_{-1}, \mathfrak{g}_{0}\right)$ and denoted by $H_{\left(\mathfrak{g}_{-1}, \mathfrak{g}_{0}\right)_{*}}^{k, s}$.

1.1. Proposition ( $[\mathrm{St}]$ ). The order $k$ structure functions of the $G$-structure - obstructions to identification of the $k$ th infinitesimal neighborhood of the point in a manifold with a flat $G$-structure with that at a given point $m \in M-$ span, for every $m$, the space $H_{\left(\mathfrak{g}_{-1}, \mathfrak{g}_{0}\right)_{*}}^{k, 2}$. These obstructions are defined provided obstructions of lesser orders vanish.

1.2. Example. All structure functions of any $G L(n)$-structure vanish identically, so all $G L(n)$-structures are locally equivalent, in particular, locally flat. Indeed: by a theorem of Serre $(\underline{\mathrm{St}}]) H^{2}\left(V ;(V, \mathfrak{g l}(V))_{*}\right)=0$. 
Clearly, the order of the structure functions of a given $G$-structure may run 1 to $N+2$ (or 1 to $\infty$ if $N=\infty$ ), where $\left(\mathfrak{g}_{-1}, \mathfrak{g}_{0}\right)_{*}=\underset{i=-1}{\bigoplus} \mathfrak{g}_{i}$.

1.3. Example. Let $\mathfrak{g}_{0}=\mathfrak{c o}(V):=\mathfrak{o}(V) \oplus \mathbb{C} z$ be the Lie algebra of conformal transformations, $\mathfrak{g}_{-1}=V$, $\operatorname{dim} V=n$. For $n=2$, let $V=V_{1} \oplus V_{2}$ with basis $\partial_{x}$ and $\partial_{y}$ and let $\mathfrak{o}(V):=\mathbb{C}\left(x \partial_{x}-y \partial_{y}\right)$. Then (Liouville's theorem, [St]

$$
(V, \mathfrak{c o}(V))_{*}=\left\{\begin{array}{ll}
\mathfrak{v e c t}\left(V^{*}\right) & \text { for } n=1, \\
\mathfrak{v e c t}\left(V_{1}^{*}\right) \oplus \mathfrak{v e c t}\left(V_{2}^{*}\right) & \text { for } n=2, \\
V \oplus \mathfrak{c o}(V) \oplus V^{*} \simeq \mathfrak{o}(n+2) & \text { for } n>2,
\end{array} \quad(V, \mathfrak{o}(V))_{*}= \begin{cases}V & \text { for } n=1, \\
V \oplus \mathfrak{o}(V) & \text { for } n \geq 2 .\end{cases}\right.
$$

The values of the Riemann tensor on any $n$-dimensional Riemannian manifold belong to $H_{(V, \mathfrak{o}(V))_{*}}^{2,2}$ whereas $H_{(V, \mathfrak{o}(V))_{*}}^{1,2}=0$.

The fact that $H_{(V, \mathfrak{o}(V))_{*}}^{1,2}=0$ (no torsion) is usually referred to as (a part of) the Levi-Civita theorem. It implies that, in the Taylor series expansion of the metric at some point (here $\eta$ is the canonical form; $x$ is the vector of coordinates, so $x^{2}$ is the vector of pairs of coordinates, etc.),

$$
g(x)=\eta+s_{1} x+s_{2} x^{2}+s_{3} x^{3}+\ldots
$$

the term $s_{1}$ can be eliminated by a choice of coordinates. Since there are no structure functions of orders $>2$, all the $s_{i}$ with $i \geq 2$ only depend on the Riemann tensor.

1.4. Remark. (cf. [Go.) Let $H_{k}^{s}$ be the degree $k$ component of $H^{s}\left(\mathfrak{g}_{-1} ;\left(\mathfrak{g}_{-1}, \mathfrak{g}_{0}\right)_{*}\right)$ with respect to the $\mathbb{Z}$-grading induced by the $\mathbb{Z}$-grading of $\left(\mathfrak{g}_{-1}, \mathfrak{g}_{0}\right)_{*}$. Clearly, $H_{\left(\mathfrak{g}_{-1}, \mathfrak{g}_{0}\right)_{*}}^{k, s}=H_{k}^{s}$, so

$$
\underset{k}{\oplus} H_{\left(\mathfrak{g}_{-1}, \mathfrak{g}_{0}\right)_{*}}^{k, s}=H^{s}\left(\mathfrak{g}_{-1} ;\left(\mathfrak{g}_{-1}, \mathfrak{g}_{0}\right)_{*}\right) .
$$

This remark considerably simplifies calculations, in particular, if the Lie algebra $\left(\mathfrak{g}_{-1}, \mathfrak{g}_{0}\right)_{*}$ is simple and finite dimensional, we can apply the BWB theorem. In the nonholonomic case considered in what follows we apply the remark to give a compact definition ${ }^{3}$ of structure functions. We can recover the bigrading at any moment but to work with just one grading is much simpler.

\section{§2. Structure FunCtions of nonholonomic StruCtures}

To embrace contact-like structures, we have to slightly generalize the notion of Cartan prolongation: with the tangent bundle over every nonholonomic manifold there is naturally associated a bundle of graded nilpotent Lie algebras, cf. $\mathrm{VG}, \mathrm{M}$. For example, for any odd dimensional manifolds with a contact structure, this is a bundle of Heisenberg Lie algebras.

2.1. Nonholonomic manifolds (VG, VG2 ). Nonholonomic manifolds. TanakaShchepochkina prolongs. Let $M^{n}$ be an $n$-dimensional manifold with a nonintegrable distribution $\mathcal{D}$. Let

$$
\mathcal{D}=\mathcal{D}_{-1} \subset \mathcal{D}_{-2} \subset \mathcal{D}_{-3} \cdots \subset \mathcal{D}_{-d}
$$

be the sequence of strict inclusions, where the fiber of $\mathcal{D}_{-i}$ at a point $x \in M$ is

$$
\mathcal{D}_{-i+1}(x)+\left[\mathcal{D}_{-1}, \mathcal{D}_{-i+1}\right](x)
$$

(here $\left.\left[\mathcal{D}_{-1}, \mathcal{D}_{-i-1}\right]=\operatorname{Span}\left([X, Y] \mid X \in \Gamma\left(\mathcal{D}_{-1}\right), Y \in \Gamma\left(\mathcal{D}_{-i-1}\right)\right)\right)$ and $d$ is the least number such that

$$
\mathcal{D}_{-d}(x)+\left[\mathcal{D}_{-1}, \mathcal{D}_{-d}\right](x)=\mathcal{D}_{-d}(x) .
$$

\footnotetext{
${ }^{3}$ Cf. with the problems encountered in the pioneer papers [T], where only $d=2$ is considered. Wagner's tensors (for any $d$ ) look even more horrible, see [DG], Va6].
} 
In case $\mathcal{D}_{-d}=T M$ the distribution is called completely nonholonomic. The number $d=$ $d(M)$ is called the nonholonomicity degree. A manifold $M$ with a distribution $\mathcal{D}$ on it will be referred to as nonholonomic one if $d(M) \neq 1$. Let

$$
n_{i}(x)=\operatorname{dim} \mathcal{D}_{-i}(x) ; \quad n_{0}(x)=0 ; \quad n_{d}(x)=n-n_{d-1} .
$$

The distribution $\mathcal{D}$ is said to be regular if all the dimensions $n_{i}$ are constants on $M$. We will only consider regular, completely nonholonomic distributions, and, moreover, satisfying certain transitivity condition (??) introduced below.

To the tangent bundle over a nonholonomic manifold $(M, \mathcal{D})$ we assign a bundle of $\mathbb{Z}$ graded nilpotent Lie algebras as follows. Fix a point $p t \in M$. The usual adic filtration by powers of the maximal ideal $\mathfrak{m}:=\mathfrak{m}_{p t}$ consisting of functions that vanish at $p t$ should be modified because distinct coordinates may have distinct "degrees". The distribution $\mathcal{D}$ induces the following filtration in $\mathfrak{m}$ :

$$
\begin{aligned}
\mathfrak{m}_{k}= & \left\{f \in \mathfrak{m} \mid X_{1}^{a_{1}} \ldots X_{n}^{a_{n}}(f)=0 \text { for any } X_{1}, \ldots, X_{n_{1}} \in \Gamma\left(\mathcal{D}_{-1}\right),\right. \\
& X_{n_{1}+1}, \ldots, X_{n_{2}} \in \Gamma\left(\mathcal{D}_{-2}\right), \ldots, X_{n_{d-1}+1}, \ldots, X_{n} \in \Gamma\left(\mathcal{D}_{-d}\right) \\
& \text { such that } \left.\sum_{1 \leq i \leq d} i \sum_{n_{i-1}<j \leq n_{i}} a_{j} \leq k\right\}
\end{aligned}
$$

where $\Gamma\left(\mathcal{D}_{-j}\right)$ is the space of germs at $p t$ of sections of the bundle $\mathcal{D}_{-j}$. Now, to a filtration

$$
\mathcal{D}=\mathcal{D}_{-1} \subset \mathcal{D}_{-2} \subset \mathcal{D}_{-3} \cdots \subset \mathcal{D}_{-d}=T M,
$$

we assign the associated graded bundle

$$
\operatorname{gr}(T M)=\oplus \operatorname{gr} \mathcal{D}_{-i}, \quad \text { where } \operatorname{gr} \mathcal{D}_{-i}=\mathcal{D}_{-i} / \mathcal{D}_{-i+1}
$$

and the bracket of sections of $\operatorname{gr}(T M)$ is, by definition, the one induced by bracketing vector fields, the sections of $T M$. We assume a "transitivity condition": The Lie algebras

$$
\left.\operatorname{gr}(T M)\right|_{p t}
$$

induced at each point $p t \in M$ are isomorphic.

The grading of the coordinates determines a nonstandard grading of $\mathfrak{v e c t}(n)$ (recall (??)):

$$
\begin{aligned}
& \operatorname{deg} x_{1}=\ldots=\operatorname{deg} x_{n_{1}}=1, \\
& \operatorname{deg} x_{n_{1}+1}=\ldots=\operatorname{deg} x_{n_{2}}=2, \\
& \ldots \ldots \ldots \ldots \ldots \ldots \ldots \ldots \ldots \ldots \\
& \operatorname{deg} x_{n-n_{d-1}+1}=\ldots=\operatorname{deg} x_{n}=d .
\end{aligned}
$$

Denote by $\mathfrak{v}=\underset{i \geq-d}{\oplus} \mathfrak{v}_{i}$ the algebra $\mathfrak{v e c t}(n)$ with the grading (2.4). One can show that the "complete prolong" of $\mathfrak{g}_{-}$to be defined shortly, i.e., $\left(\mathfrak{g}_{-}\right)_{*}:=\left(\mathfrak{g}_{-}, \tilde{\mathfrak{g}}_{0}\right)_{*} \subset \mathfrak{v}$, where $\tilde{\mathfrak{g}}_{0}:=\mathfrak{d} \mathfrak{e} \mathfrak{r}_{0} \mathfrak{g}_{-}$, preserves $\mathcal{D}$.

For nonholonomic manifolds, an analog of the group $G$ from the term " $G$-structure", or rather of its Lie algebra, $\mathfrak{g}=\operatorname{Lie}(G)$, is the pair $\left(\mathfrak{g}_{-}, \mathfrak{g}_{0}\right)$, where $\mathfrak{g}_{0}$ is a subalgebra of the $\mathbb{Z}$-grading preserving Lie algebra of derivations of $\mathfrak{g}_{-}$, i.e., $\mathfrak{g}_{0} \subset \mathfrak{d} \mathfrak{e} \mathfrak{r}_{0} \mathfrak{g}_{-}$. If $\mathfrak{g}_{0}$ is not explicitly indicated, we assume that $\mathfrak{g}_{0}=\mathfrak{d} \mathfrak{e} \mathfrak{r}_{0} \mathfrak{g}_{-}$, i.e., is the largest possible.

Given a pair $\left(\mathfrak{g}_{-}, \mathfrak{g}_{0}\right)$ as above, define its Tanaka-Shchepochkina prolong to be the maximal subalgebra $\left(\mathfrak{g}_{-}, \mathfrak{g}_{0}\right)_{*}=\underset{k \geq-d}{\bigoplus} \mathfrak{g}_{k}$ of $\mathfrak{v}$ with given non-positive part $\left(\mathfrak{g}_{-}, \mathfrak{g}_{0}\right)$. For an explicit construction of the components, see [Sh14, [Y], ShN] and below. 
Natural bases in $T_{m} M$ : the $D_{i}$ 's and the $Q_{i}$ 's ([ShN]). Vershik and Gershkovich showed VG] that every nonholonomic structure $\mathcal{D}$ on $M$ determines a structure of $\mathbb{Z}$-graded nilpotent Lie algebra in $\operatorname{gr}(T M)$. We will only consider manifolds with a transitive action of the diffeomorphism group of $M$, i.e., the manifolds for which these Lie algebras are isomorphic.

A natural basis in every tangent space $T_{m} M$ to any manifold $M$ is given by partial derivatives. If $M$ is endowed with a nonholonomic structure, then there are two types of natural bases in gr $T_{m} M$. In physics literature on supersymmetry and supergravity, the elements of these two bases that generate the Lie algebra gr $T_{m} M$ are denoted the $D_{i}$ 's and the $Q_{i}$ 's, respectively.

Let us consider the simplest example. Let $\operatorname{dim} M=2 n+1$ and let the nonholonomic structure on $M$ be given by the contact form $\alpha=d t-\sum\left(p_{i} d q_{i}-q_{i} d p_{i}\right)$. The vector fields that belong to the distribution $\mathcal{D}$ are the fields

$$
X=f \partial_{t}+\sum\left(g_{i} \partial_{q_{i}}+h_{i} \partial_{p_{i}}\right) \text { such that } \alpha(X)=f-\sum\left(p_{i} g_{i}+q_{i} h_{i}\right)=0 .
$$

In particular, we see that neither $\partial_{q_{i}}$ nor $\partial_{p_{i}}$ belongs to $\mathcal{D}$, but rather

$$
D_{p_{i}}=\partial_{q_{i}}+p_{i} \partial_{t} \text { and } D_{q_{i}}=\partial_{p_{i}}-q_{i} \partial_{t}
$$

These $D_{p_{i}}$ and $D_{q_{i}}$ are examples of the $D$-type basis vectors. They, and their brackets, span the space of sections of $\operatorname{gr}(T M)$ at any given point $m$. By abuse of speech, we say that the $D$-vectors span $T_{m} M$, and same applies to $Q$-vectors defined below.

Now, the Lie algebra that preserves $\mathcal{D}$ consists of vector fields $X$ such that (here $L_{X}$ is the Lie derivative along $X$ )

$$
L_{X}(\alpha)=0
$$

The corresponding vector fields in our particular case of the contact distribution are contact vector fields $K_{f}$ generated by $f \in \mathbb{C}[t, p, q]$ :

$$
K_{f}=(2-E)(f) \frac{\partial}{\partial t}-H_{f}+\frac{\partial f}{\partial t} E
$$

where $E=\sum_{i} y_{i} \frac{\partial}{\partial y_{i}}$ (here the $y_{i}$ are all the coordinates except $t$ ) is the Euler operator, and $H_{f}$ is the Hamiltonian field with Hamiltonian $f$ that preserves $d \alpha$ :

$$
H_{f}=\sum_{i \leq n}\left(\frac{\partial f}{\partial p_{i}} \frac{\partial}{\partial q_{i}}-\frac{\partial f}{\partial q_{i}} \frac{\partial}{\partial p_{i}}\right) \text {. }
$$

(As is easy to see,

$$
L_{K_{f}}(\alpha)=2 \frac{\partial f}{\partial t} \alpha
$$

where $L_{X}$ is the Lie derivative along $X$.) The basis of the tangent space is spanned by

$$
K_{p_{i}}=\partial_{q_{i}}-p_{i} \partial_{t} \text { and } K_{q_{i}}=\partial_{p_{i}}+q_{i} \partial_{t}
$$

and their brackets. These $K_{p_{i}}$ and $K_{q_{i}}$ are examples of the $Q$-type basis vectors.

How to interpret the $D$-type and the $Q$-type vectors? Let

$$
\mathfrak{n}=\underset{-d \leq i \leq-1}{\oplus} \mathfrak{n}_{i}
$$

be a nilpotent Lie algebra generated by $\mathfrak{n}_{-1}$. Let $B=\left\{b_{1}, \ldots, b_{n}\right\}$ be a graded basis of $\mathfrak{n}$ (the basis is said to be graded if its first $n_{1}:=\operatorname{dim} \mathfrak{n}_{-1}$ elements span $\mathfrak{g}_{-1}$, the next $n_{2}:=\operatorname{dim} \mathfrak{n}_{-2}$ elements span $\mathfrak{n}_{-2}$, and so on). Let $N$ be the connected and simply connected Lie group 
with the Lie algebra $\mathfrak{n}$. On $N$, consider the two systems of vector fields: the left-invariant fields $D_{i}$ and the right-invariant fields $Q_{i}$ such that ( $e$ is the unit of $N$ )

$$
D_{i}(e)=Q_{i}(e)=b_{i} \text { for all } i=1, \ldots, n .
$$

NB: Here we deviate from the conventions of physical papers where the symbols $D_{i}$ and $Q_{i}$ are only applied to the generators of $\mathfrak{n}$, i.e., to the first $n_{1}$ elements.

Let $\mathfrak{g}_{-}$be a realization of $\mathfrak{n}$ by left-invariant vector fields, so the vectors $D_{i}(e)$ span $\mathfrak{g}_{-}$. Let $\theta^{i}$ be right-invariant 1 -forms on $N$ such that

$$
\theta^{i}\left(Q_{j}\right)=\delta_{j}^{i}
$$

Now, any vector field $X$ on $N$ is of the form

$$
X=\sum_{i=1}^{n} \theta^{i}(X) Q_{i} .
$$

Since each $D_{i}$ commutes with each $Q_{j}$ (if $\mathfrak{n}$ is a Lie superalgebra, they supercommute), it follows that

$$
\theta^{i}\left(\left[D_{j}, X\right]\right)=D_{j}\left(\theta^{i}(X)\right) .
$$

Now, let us determine a right-invariant distribution $\mathcal{D}$ on $N$ such that $\left.\mathcal{D}\right|_{e}=\mathfrak{n}_{-1}$. Clearly, $\mathcal{D}$ is singled out in $T N$ by eqs. for $X \in \mathfrak{v e c t}(n)$

$$
\theta^{n_{1}+1}(X)=0, \quad \ldots, \quad \theta^{n}(X)=0 .
$$

Since each $D_{i}$ commutes with each $Q_{j}$, the algebra $\mathfrak{g}_{-}$preserves $\mathcal{D}$. The coordinates $(2.4)$ on $N$ described above determine two embeddings of $\mathfrak{n} \operatorname{into} \mathfrak{v e c t}(n)$ : one is spanned by the $D_{i}$ and the other one by the $Q_{i}$.

Denote by $\mathfrak{g}=\underset{i>-d}{\oplus} \mathfrak{g}_{i}$ the algebra $\mathfrak{v e c t}(n)$ with the grading (??). Then $\mathfrak{g}_{-}=\underset{i<0}{\bigoplus_{i}} \mathfrak{g}_{i}$ preserves $\mathcal{D}$. We will show later that the "complete prolongation" of $\mathfrak{g}_{-}$, i.e., $\left(\mathfrak{g}_{-}\right)_{*}:=\left(\mathfrak{g}_{-}, \tilde{\mathfrak{g}}_{0}\right)_{*}$, where $\tilde{\mathfrak{g}}_{0}:=\mathfrak{d} \mathfrak{e} \mathfrak{r}_{0} \mathfrak{g}_{-}$, also preserves $\mathcal{D}$.

Thus we see that, with every nonholonomic manifold $(M, \mathcal{D})$, a natural $G$-structure is associated, its Lie algebra is $\operatorname{Lie}(G)=\mathfrak{d} \mathfrak{e r}_{0} \mathfrak{g}_{-}$. But the structure functions of this $G$-structure do not reflect the nonholonomic nature of $M$.

Indeed, recall an example from [St]. Let $W_{1} \subset W$ be a subspace of dimension $k$ and $G \subset G L(W)$ the parabolic subgroup that preserves the subspace. Then to determine a $G$-structure on $M$, where $\operatorname{dim} M=\operatorname{dim} W$, is the same as to determine a differential $k$ system or a $k$-dimensional distribution. A fixed frame $f$ in $T_{m} M$ determines an isomorphism $f: W \longrightarrow T_{m} M$. Given a $G$-structure on $M$, we set $\mathcal{D}(m)=f\left(W_{1}\right)$. Since $G$ preserves $W_{1}$, the subspace $\mathcal{D}(m)$ indeed depends only on $m$, not on $f$.

The other way round, given a distribution $\mathcal{D}$, consider the frames $f$ such that $f^{-1}(\mathcal{D}(m))=$ $W_{1}$. They form a $G$-structure. The flat $G$-structures correspond to integrable distributions.

To take the nonholonomic nature of $M$ into account, we need something new — an analog of the above Proposition 1.1 for the case where the natural basis of the tangent space consists not of partial derivatives but rather of covariant derivatives corresponding to the connection determined by the same Pfaff equations that determine the distribution, and therefore instead of $T_{m} M=\mathfrak{g}_{-1}$ we have $(\operatorname{gr}(T M))_{m}=\mathfrak{g}_{-}$. To be able to formulate such Proposition, we have to define (1) the simplest nonholonomic structure - the "flat" one,

(2) the analog of $\mathfrak{g}_{0}$ when $\mathfrak{g}_{-1}$ is replaced by $\mathfrak{g}_{-}$and only distribution is given,

(3) what is the analog of $\left(\mathfrak{g}_{-1}, \mathfrak{g}_{0}\right)_{*}$,

(4) what is the analog of $H_{\left(\mathfrak{g}_{-1}, \mathfrak{g}_{0}\right)_{*}}^{k,}$.

Here are the answers: 
1) Let $\mathcal{D}$ be a nonholonomic distribution in $M$, let $F$ be the flag which $\mathcal{D}$ determines at a point $m \in M$. Let $N:=\mathbb{K}^{n}$ with a fixed flag $F$ and a fixed frame $f$. Having identified $T_{n} N$ with $N$ by means of the translation by $n$ considered as an element of the nilpotent Lie group $N$ whose Lie algebra is $\mathfrak{g}_{-}$(since the group $N$ is not commutative now, we select, say, left translations) we fix a frame and a flag - the images of $f$ and $F$ - in each $T_{n} N$. A flat nonholonomic structure on $N$ is the pair of bundles (the frame bundle, the distribution $\mathcal{D}$ ); the fibers of both bundles over $n$ are obtained from the fixed frame and flag, respectively, by means of the $G$-action, where $G$ is the (connected and simply connected) Lie group whose Lie algebra $\mathfrak{g}_{0}$ is defined at the next step.

2) If only a distribution $\mathcal{D}$ is given, we set $\mathfrak{g}_{0}:=\mathfrak{d} \mathfrak{e} \mathfrak{r}_{0} \mathfrak{g}_{-}$; it is often interesting to consider an additional structure on the distribution, say Riemannian, cf. VG2, as in the case of Carnot-Carathéodory metric in which case $\mathfrak{g}_{0}$ is a subalgebra of $\mathfrak{d} \mathfrak{e r}_{0} \mathfrak{g}_{-}$, e.g., $\mathfrak{d} \mathfrak{r r}_{0} \mathfrak{g}_{-} \cap \mathfrak{o}\left(\mathfrak{g}_{-1}\right)$.

3) Given a pair $\left(\mathfrak{g}_{-}, \mathfrak{g}_{0}\right)$ as above, define its $k$ th Tanaka-Shchepochkina prolong (given simultaneously, although [Sh1] was published later than [T]; Sh1] also embraces Lie superalgebras and various partial prolongs, see [Sh2]) for $k>0$ to be:

$$
\mathfrak{g}_{k}=\left(i\left(S^{\bullet}\left(\mathfrak{g}_{-}^{*}\right) \otimes \mathfrak{g}_{0}\right) \cap j\left(S^{\bullet}\left(\mathfrak{g}_{-}^{*}\right) \otimes \mathfrak{g}_{-}\right)\right)_{k},
$$

where the subscript singles out the component of degree $k$, where $S^{\bullet}=\oplus S^{i}$ and $S^{i}$ denotes the operator of the $i$ th symmetric power, and where

$$
\begin{aligned}
& i: S^{k+1}\left(\mathfrak{g}_{-1}^{*}\right) \otimes \mathfrak{g}_{-1} \longrightarrow S^{k}\left(\mathfrak{g}_{-1}^{*}\right) \otimes \mathfrak{g}_{-1}^{*} \otimes \mathfrak{g}_{-1}, \\
& j: S^{k}\left(\mathfrak{g}_{-1}^{*}\right) \otimes \mathfrak{g}_{0} \longrightarrow S^{k}\left(\mathfrak{g}_{-1}^{*}\right) \otimes \mathfrak{g}_{-1}^{*} \otimes \mathfrak{g}_{-1}
\end{aligned}
$$

are natural embeddings.

Similarly to the case where $\mathfrak{g}_{-}$is commutative, define $\left(\mathfrak{g}_{-}, \mathfrak{g}_{0}\right)_{*}$ to be $\underset{k \geq-d}{\oplus} \mathfrak{g}_{k}$ with $\mathfrak{g}_{k}$ for $k>0$ given by $(2.10)$; then, as is easy to verify, $\left(\mathfrak{g}_{-}, \mathfrak{g}_{0}\right)_{*}$ is a Lie algebra.

4) Arguments similar to those of [St] should show that $H^{2}\left(\mathfrak{g}_{-} ;\left(\mathfrak{g}_{-}, \mathfrak{g}_{0}\right)_{*}\right)$ is the space of values of all nonholonomic structure functions - obstructions to the identification of the infinitesimal neighborhood of a point $m$ of the manifold $M$ with a nonholonomic structure (given by $\mathfrak{g}_{-}$and $\mathfrak{g}_{0}$ ) with the infinitesimal neighborhood of a point of a flat nonholonomic manifold with the same $\mathfrak{g}_{-}$and $\mathfrak{g}_{0}$. We intend to give a detailed proof of this statement elsewhere.

The space $H^{2}\left(\mathfrak{g}_{-} ;\left(\mathfrak{g}_{-}, \mathfrak{g}_{0}\right)_{*}\right)$ naturally splits into homogeneous components whose degrees will be called the orders of the structure functions; the orders run $2-d$ to $N+2 d$ (or to $\infty$ if $N=\infty)$. As in the case of a commutative $\mathfrak{g}_{-}=\mathfrak{g}_{-1}$, the structure functions of order $k$ can be interpreted as obstructions to flatness of the nonholonomic manifold with the $\left(\mathfrak{g}_{-}, \mathfrak{g}_{0}\right)$ structure provided the obstructions of lesser orders vanish. Observe that, for nonholonomic manifolds, the order of structure functions is no more in direct relation with the orders of the infinitesimal neighborhoods of the points we wish to identify: distinct partial derivatives bear different "degrees".

Different filtered algebras $L$ with the same graded $\mathfrak{g}_{-}$are governed precisely by the coboundaries responsible for filtered deformations of $\mathfrak{g}_{-}$, and all of them vanish in cohomology, so the above nonholonomic structure functions are well-defined.

\section{§3. The Riemann and Weyl tensors. Projective structures}

The conformal case. For the classical domains $X=S / P$ that Goncharov considered, the structure functions are generalizations of the Weyl tensor - the conformally invariant part 
of the Riemann tensor (the case $S=O(n+2)$ and $G=C O(n)$ ). In most of these cases

$$
\left(\mathfrak{g}_{-1}, \mathfrak{g}_{0}\right)_{*}=\mathfrak{s}(:=\operatorname{Lie}(S))
$$

and the description of the structure functions is a particular case of the BWB theorem. In particular, if $(3.1)$ holds, the space $H^{2}\left(\mathfrak{g}_{-1} ;\left(\mathfrak{g}_{-1}, \mathfrak{g}_{0}\right)_{*}\right)$, considered as a $\mathfrak{g}_{0}$-module, has the same number of irreducible components and the same dimension as $E^{2}\left(\mathfrak{g}_{-1}\right)$; only weights differ.

The generalized Riemannian case. When we reduce $\mathfrak{g}_{0}$, by retaining its semi-simple part $\hat{\mathfrak{g}_{0}}$ and deleting the center, we can not directly apply the BWB theorem because $\left(\mathfrak{g}_{-1}, \hat{\mathfrak{g}_{0}}\right)_{*}=$ $\mathfrak{g}_{-1} \oplus \hat{\mathfrak{g}}_{0}$ is not simple but we can reduce teh problem to the conformal case, since, as is known,

$$
H^{2}\left(\mathfrak{g}_{-1} ;\left(\mathfrak{g}_{-1}, \hat{\mathfrak{g}_{0}}\right)_{*}\right)=H^{2}\left(\mathfrak{g}_{-1} ; \mathfrak{s}\right) \oplus S^{2}\left(\mathfrak{g}_{-1}^{*}\right) .
$$

For the nonholonomic case, a similar reduction is given by Premet's theorem (below). Its general case, though sufficiently neat, is not as simple as (3.2). However, although the following analog of (3.2) is not always true

$$
H^{2}\left(\mathfrak{g}_{-} ;\left(\mathfrak{g}_{-}, \hat{\mathfrak{g}_{0}}\right)_{*}\right)=H^{2}\left(\mathfrak{g}_{-} ; \mathfrak{s}\right) \oplus S^{2}\left(\mathfrak{g}_{-1}^{*}\right),
$$

it is still true in many cases of interest: for the "contact grading".

The projective case. Theorems of Serre and Yamaguchi. When (3.1) fails, $\mathfrak{s}$ is a proper subalgebra of $\left(\mathfrak{g}_{-1}, \mathfrak{g}_{0}\right)_{*}$. It is of interest therefore

(a) to list all the cases where, having started from a simple Lie (super)algebra $\mathfrak{s}=\underset{i \geq-d}{\oplus} \mathfrak{s}_{i}$, we have the following analog of (3.1)

$$
\left(\mathfrak{s}_{-}, \mathfrak{s}_{0}\right)_{*}=\mathfrak{s}
$$

and

(b) find out what is the "complete prolongation" of $\mathfrak{s}_{-}$, i.e., what is $\left(\mathfrak{s}_{-}\right)_{*}:=\left(\mathfrak{s}_{-}, \tilde{\mathfrak{s}}_{0}\right)_{*}$, where $\tilde{\mathfrak{s}}_{0}:=\mathfrak{d} \mathfrak{e r}_{0} \mathfrak{s}_{-}$.

For the simple finite dimensional Lie algebras $\mathfrak{s}$, Yamaguchi $[\underline{Y}$ gives the answer. It is rather interesting and we reproduce it. The answer for simple Lie superalgebras is obtained by Shchepochkina (unpublished). Comment: one would expect that $\tilde{\mathfrak{s}}_{0}$ strictly contains $\mathfrak{s}_{0}$, and hence $\left(\mathfrak{s}_{-}\right)_{*}$ should strictly contain $\mathfrak{s}$; instead they are equal (in particular, $\tilde{\mathfrak{s}}_{0}=\mathfrak{s}_{0}$ ).

Theorem $([\mathrm{Y}])$. Equality $\left(\mathfrak{s}_{-}\right)_{*}=\mathfrak{s}$ holds almost always. The exceptions are

1) $\mathfrak{s}$ with the grading of depth $d=1$ (in which case $\left(\mathfrak{s}_{-}\right)_{*}=\mathfrak{v e c t}\left(\mathfrak{s}_{-}^{*}\right)$ );

2) $\mathfrak{s}$ with the grading of depth $d=2$ and $\operatorname{dim} \mathfrak{s}_{-2}=1$, i.e., with the "contact" grading, in which case $\left(\mathfrak{s}_{-}\right)_{*}=\mathfrak{k}\left(\mathfrak{s}_{-}^{*}\right)$ (these cases correspond to exclusion of the nodes on the Dynkin graph connected with the node for the maximal root on the extended graph);

$3) \mathfrak{s}$ is either $\mathfrak{s l}(n+1)$ or $\mathfrak{s p}(2 n)$ with the grading determined by "selecting" the first and the $i$ th of simple coroots, where $1<i<n$ for $\mathfrak{s l}(n+1)$ and $i=n$ for $\mathfrak{s p}(2 n)$. (Observe that, in this case, $d=2$ with $\operatorname{dim} \mathfrak{s}_{-2}>1$ for $\mathfrak{s l}(n+1)$ and $d=3$ for $\mathfrak{s p}(2 n)$.)

Moreover, $\left(\mathfrak{s}_{-}, \mathfrak{s}_{0}\right)_{*}=\mathfrak{s}$ is true almost always. The cases where this fails (the ones where a projective action is possible) are $\mathfrak{s l}(n+1)$ or $\mathfrak{s p}(2 n)$ with the grading determined by "selecting" only one (the first) simple coroot.

Case 1) of Yamaguchi's theorem: for the conformal (Weyl) case, see [Go] for the Riemannian case, see [LPS] .

For the classical domains $X=S / P,(3.1)$ fails only for $S=S L(n+1)$ and $X=\mathbb{C} P^{n}$; in this case $\mathfrak{g}_{0}=\mathfrak{g l}(n)$ and $\left(\mathfrak{g}_{-1}, \mathfrak{g}_{0}\right)_{*}=\mathfrak{v e c t}(n)$, the Lie algebra of vector fields in $n$ indeterminates. The space of "total" structure functions $H^{2}\left(\mathfrak{g}_{-1} ; \mathfrak{v e c t}(n)\right)$ differs from $H^{2}\left(\mathfrak{g}_{-1} ; \mathfrak{s}\right)$, the latter 
structure functions correspond to obstructions to the projective structure. For many facets of projective structures, see $\mathrm{OT}$ and $\mathrm{BR}$.

The Riemannian version of this projective case, corresponds to $\hat{\mathfrak{g}_{0}}=\mathfrak{s l}(n)$ and $\left(\mathfrak{g}_{-1}, \hat{\mathfrak{g}_{0}}\right)_{*}=$ $\mathfrak{s v e c t}(n)$, the Lie algebra of divergence free vector fields.

The cases of "complete prolongation" $\left(\mathfrak{s}_{-1}\right)_{*}=\mathfrak{v} \mathfrak{e c t}\left(\mathfrak{s}_{-1}^{*}\right)$ and their "Riemannian version" $\left(\mathfrak{s}_{-1}\right)_{*}=\mathfrak{s v e c t}\left(\mathfrak{s}_{-1}^{*}\right)$, as well as $\left(\mathfrak{s}_{-1}\right)_{*}=\mathfrak{h}\left(\mathfrak{s}_{-1}^{*}\right)$, were considered by Serre long ago, see [St], and the answer is as follows:

Theorem (Serre, see [St]; for super version, see [LPS] and GLS]).

1) $H^{2}\left(\mathfrak{s}_{-1} ; \mathfrak{v e c t}(n)\right)=0$ and $H^{2}\left(\mathfrak{s}_{-1} ; \mathfrak{s v e c t}(n)\right)=0$.

2) $H^{2}\left(\mathfrak{s}_{-1} ; \mathfrak{h}(2 n)\right)=E^{3}\left(\mathfrak{s}_{-1}^{*}\right)$.

Case 2) of Yamaguchi's theorem is taken care of by one of Premet's theorems and formula (3.5) below.

Case 3) of Yamaguchi's theorem is done in $\S 6$ of this paper.

$\overline{\text { In what follows, for manifolds } X}=S / P$ with nonholonomic structure, we say "N-Weyl" or "N-conformal", for tensors corresponding to cohomology of $\mathfrak{g}_{-}$with coefficients in $\left(\mathfrak{g}_{-}, \mathfrak{g}_{0}\right)_{*}$, "N-Riemannian" for nonholonomic structure functions $\left(\mathfrak{g}_{-}, \hat{\mathfrak{g}_{0}}\right)_{*}$, where $\hat{\mathfrak{g}_{0}}$ is the semi-simple part of $\mathfrak{g}_{0}$, and "N-projective" for the coefficients in $\mathfrak{s}=\operatorname{Lie}(S)$ whenever $\mathfrak{s}$ is smaller than $\left(\mathfrak{g}_{-}, \mathfrak{g}_{0}\right)_{*}$, for example, for partial Cartan prolongs, see [LSh].

The simplest examples (exclusion of the first simple coroot of $\mathfrak{s p}(2 n+2)$ ). Let $\mathfrak{g}_{-}=\mathfrak{h} \mathfrak{e}(2 n)$, the Heisenberg Lie algebra. Then $\mathfrak{g}_{0}=\mathfrak{c} \mathfrak{s}(2 n)$ (i.e., $\mathfrak{s p}(2 n) \oplus \mathbb{C} z$ ) and $\left(\mathfrak{g}_{-}, \mathfrak{g}_{0}\right)_{*}$ is the Lie algebra $\mathfrak{k}(2 n+1)$ of contact vector fields.

So far, there is no analog of Serre's theorem on involutivity for simple $\mathbb{Z}$-graded Lie algebras of depth $>1$, cf. [LPS], and examples from [GLS] show that if exists, the theorem is much more involved.

The fact that

$$
H^{2}(\mathfrak{h} \mathfrak{e}(2 n) ; \mathfrak{k}(2 n+1))=0
$$

explains why the Pfaff equation $\alpha(X)=0$ for $X \in \mathfrak{v e c t}(2 n+1)$ can be reduced to a canonical form, cf. [Z]. This fact is an easy corollary of a statement on cohomology of coinduced modules $\left[\mathrm{FF}\right.$. For the N-Riemannian tensor in this case, we have: $\hat{\mathfrak{g}}_{0}=\mathfrak{s p}(2 n)$ and $\left(\mathfrak{g}_{-}, \hat{\mathfrak{g}_{0}}\right)_{*}$ is the Poisson Lie algebra $\mathfrak{p o}(2 n)$. The Poisson Lie algebra is spanned by fields $K_{f}$, where $\frac{\partial f}{\partial t}=0$. Now, from (3.5) and the short exact sequence

$$
0 \longrightarrow \mathfrak{p o}(2 n) \longrightarrow \mathfrak{k}(2 n+1) \stackrel{\frac{\partial}{\partial t}: K_{f} \mapsto \frac{\partial f}{\partial t}}{\longrightarrow} \mathbb{C}[t, p, q] \longrightarrow 0
$$

we easily deduce (using the corresponding long exact sequence, see $[\mathrm{FF}]$ ) that

$$
H^{2}(\mathfrak{h} \mathfrak{i}(2 n) ; \mathfrak{p o}(2 n))=0 .
$$

In our terms, this fact (usually called Darboux's theorem and proved by analytic means [Z]) is an explanation why the contact form $\alpha$ can be reduced to a canonical form not only at any point but locally.

Other examples. For numerous examples of N-projective structures in various instances, see [C1 - C5] and [YY], and (in super setting) MaG]. Armed with SuperLie, one can now easily perform the computations of relevant Lie algebra cohomology. Premet's theorems tell what to compute in the N-Riemannian case and again with SuperLie this will be easy: we just give a few samples (one selected simple coroot for every $\mathfrak{s}$ and two selected coroots for the two series of one of Yamaguchi's cases). 
§4. Premet's Theorems (from Premet's letter to DL, 10/17/1990)

In 1990, DL asked Alexander Premet: how to reduce computations of the space of values for a nonholonomic Riemann tensors to that for the nonholonomic Weyl tensor, as in (3.2)? Namely, is (3.3) always true?

Premet wrote two letters with a general answer. One letter is reproduced practically without changes below (DL is responsible for any mistakes left/inserted); it shows how to reduce the problem to computing (the 1 st) cohomology of $\mathfrak{g}_{-}$with coefficients in a certain $\mathfrak{g}_{-}-$ module which is not a $\mathfrak{g}$-module. Little was known about such cohomology except theorems of Kostant (on $H^{1}$ ) and of Leger and Luks (on $H^{2}$ ) both for the case where $\mathfrak{g}_{-}$is the maximal nilpotent subalgebra. Premet's second letter (reproduced in [LLS]) contained a mighty generalization of these theorems for $H^{i}$ for any $i$ and any $\mathfrak{g}_{-}$.

However, in nonholonomic cases, to derive an explicit answer from the BWB theorem is difficult "by hands", the extra terms in the Riemannian case (see sec. 4.4 below) add extra job. So Premet's theorems were put aside for 13 years. Now that a package SuperLie ([Gr $)$, originally designed for the purposes of supergravity, is sufficiently developed, we are able to give an explicit answer: see the next section. The cases we consider here (of the maximal parabolic subalgebras) required several minutes to compute. (But much longer to document the results, and it will require a while to interpret them, say as in [LPS.) To our regret, Premet looks at his theorems as a mere technical exercise ("a simple job for Kostant") not interesting enough to co-author the paper.

4.1. Terminological conventions. Let $\mathfrak{g}$ be a simple (finite dimensional) Lie algebra. Let $L^{\lambda}$ denote the irreducible (finite dimensional) $\mathfrak{g}$-module with the highest weight $\lambda$; let $E_{\mu}$ be the subspace the module $E$ of weight $\mu$.

Let $R$ be the root system of $\mathfrak{g}$ and $B$ the base (system of simple roots). Let $W=W(R)$ be the Weyl group of $\mathfrak{g}$ and $l(w)$ the length of the element $w \in W$; let $W_{i}$ be the subset of elements of length $i$. Let $R_{I} \subset R$ and let $B_{I}$ be the base of $R_{I}$. Set (this is a definition of $k(i)$ as well)

$$
W(I)_{i}=\left\{w_{i, 1}, \ldots, w_{i, k(i)} \in W_{i} \mid w_{i, j}^{-1}\left(B \backslash B_{I}\right)>0 \text { for all } 1 \leq j \leq k(i)\right\} .
$$

Let the Dynkin graph of $B$ be, for example, as follows:

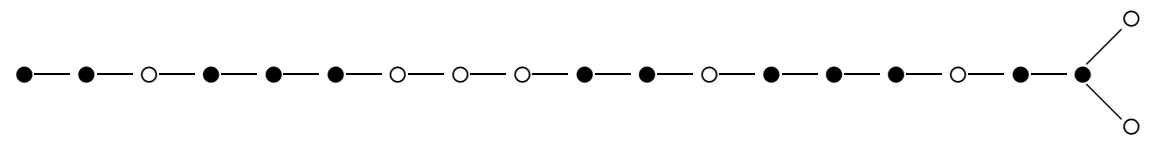

and let $B_{I}$ consist of roots corresponding to the black nodes. Let us represent $B_{I}$ as the union of connected subgraphs:

$$
B_{I}=B_{I}^{(1)} \coprod \ldots \coprod B_{I}^{(s)}
$$

where $s$ (in our example $s=5$ ) is the number of connected components of the Dynkin graph $D_{I}$ of $B_{I}$ and where $B_{I}^{(i)}$ corresponds to the $i$ th connected components of $D_{I}$ (counted from left to right). Set

$$
c=\operatorname{card} B, \quad c_{i}=\operatorname{card}\left\{\alpha \in B \backslash B_{I} \mid\left(\alpha, B_{I}^{(i)}\right) \neq 0\right\}-1 .
$$

Clearly, if $B_{I} \neq B$, then $c_{i} \in\{0,1,2\}$. For example, for the graph of $\mathfrak{o}(20)$ depicted above, we have:

$$
c=20, \quad c_{1}=0, \quad c_{2}=1, \quad c_{3}=1, \quad c_{4}=1, \quad c_{5}=2 .
$$

The following statement is obvious. 
Statement. 1) $c_{i}=2$ if and only if $R$ is of type $D_{n}, E_{6}, E_{7}, E_{8}$, one of the endpoints of $D_{I}^{(i)}$ is a branching point for $D$, and the remaining endpoint of $D_{I}^{(i)}$ is not an endpoint of $D$.

2) $c_{i}=0$ if and only if all but one of the end vertices of the graph of $B_{I}^{(i)}$ are the end vertices for the graph of $R$.

4.2. The Borel-Weil-Bott theorem. Let $\mathrm{rkg}=r>1, I \subset\{1, \ldots, r\}$; let $\mathfrak{p}=\mathfrak{p}_{I}$ be a parabolic subalgebra generated by the Chevalley generators $X_{i}^{ \pm}$of $\mathfrak{g}$ except the $X_{i}^{+}$, where $i \in I$. As is known, $\mathfrak{p}=\mathfrak{g}_{-} \oplus \mathfrak{l}$, where $\mathfrak{l}$ is the Levi (semi-simple) subalgebra generated by all the $X_{i}^{ \pm}$, where $i \notin I$. Clearly, $\mathfrak{l}=\mathfrak{l}^{(1)} \oplus \mathfrak{z}$, where $\mathfrak{l}^{(1)}$ is the derived algebra of $\mathfrak{l}$, and $\mathfrak{z}=\mathfrak{z}(\mathfrak{l})$ is the center of $\mathfrak{l}$.

So, in terms of $\S 3, \mathfrak{g}_{0}=\mathfrak{l}, \hat{\mathfrak{g}}_{0}=\mathfrak{l}^{(1)}$.

Theorem (The BWB Theorem, see [BGG]). Let $E=L^{\lambda}$ be an irreducible (finite dimensional) $\mathfrak{g}$-module with highest weight $\lambda$. Then $H^{i}\left(\mathfrak{g}_{-} ; E\right)$ is the direct sum of $\mathfrak{l}$-modules with the lowest weights $-w_{i, j}(\lambda+\rho)+\rho$, where $w_{i, j} \in W(I)_{i}$, see $(4.1)$; each such module enters with multiplicity 1.

The BWB theorem describes (for $i=2$ ) nonholonomic analogs of Weyl tensors. Theorem 4.4 describes nonholonomic analogs of the Riemann tensors. To prove sec. 4.4 , we need the following Lemma.

4.3. Lemma. Let $E=L^{\lambda}$ be an irreducible (finite dimensional) $\mathfrak{g}$-module with highest weight $\lambda$ such that $E \simeq E^{*}$. Let $V$ be a $\mathfrak{p}$-invariant subspace in $E$ which contains $E_{-}=$ $\underset{\mu=\sum k_{i} \alpha_{i} \mid k_{i}<0}{\oplus} E_{\mu}$. Let further any weight $\mu$ of $E / V$ be of the form $\mu=-\sum a_{i} \alpha_{i}$, where $a_{i} \geq 0$.

Then, for any $i<\mathrm{rkg}$, we have the $\mathfrak{l}$-module isomorphism:

$$
H^{i}\left(\mathfrak{g}_{-} ; V\right) \simeq H^{i}\left(\mathfrak{g}_{-} ; E\right) \oplus H^{i-1}\left(\mathfrak{g}_{-} ; E / V\right) .
$$

Proof. As is well known $\left[\mathrm{FF}\right.$, with every short exact sequence of $\mathfrak{g}_{-}$-modules

$$
0 \longrightarrow V \longrightarrow E \longrightarrow E / V \longrightarrow 0
$$

there is associated the long exact cohomology sequence

$$
\begin{aligned}
& 0 \longrightarrow H^{0}\left(\mathfrak{g}_{-} ; V\right) \longrightarrow H^{0}\left(\mathfrak{g}_{-} ; E\right) \stackrel{\varphi_{0}}{\longrightarrow} H^{0}\left(\mathfrak{g}_{-} ; E / V\right) \longrightarrow \\
& H^{1}\left(\mathfrak{g}_{-} ; V\right) \longrightarrow H^{1}\left(\mathfrak{g}_{-} ; E\right) \stackrel{\varphi_{1}}{\longrightarrow} H^{1}\left(\mathfrak{g}_{-} ; E / V\right) \longrightarrow \ldots \\
& \longrightarrow H^{i}\left(\mathfrak{g}_{-} ; V\right) \longrightarrow H^{i}\left(\mathfrak{g}_{-} ; E\right) \stackrel{\varphi_{i}}{\longrightarrow} H^{i}\left(\mathfrak{g}_{-} ; E / V\right) \longrightarrow \ldots
\end{aligned}
$$

Let us prove that the weight $-w(\lambda+\rho)+\rho$ can not be a weight of the l-module $H^{i}\left(\mathfrak{g}_{-} ; E / V\right)$ for $i<\mathrm{rk} \mathfrak{g}$. Indeed, each weight of $H^{i}\left(\mathfrak{g}_{-} ; E / V\right)$, which is a quotient of a submodule of $E^{i}\left(\mathfrak{g}_{-}^{*}\right) \otimes E / V$, is of the form

$$
\gamma_{1}+\ldots+\gamma_{k}+\mu
$$

where $\gamma_{1}, \ldots, \gamma_{k}$ are distinct positive roots which do not belong to the root lattice $Q\left(B_{I}\right)$, and $\mu$ is a weight of $E$ of the form $\mu=\sum_{a_{i} \geq 0} a_{i} \alpha_{i}$.

Suppose that

$$
-w(\lambda+\rho)+\rho=\gamma_{1}+\ldots+\gamma_{k}+\mu .
$$

Then $-\mu-w(\lambda)-w(\rho)+\rho=\gamma_{1}+\ldots+\gamma_{k}$. Set

$$
R_{W}^{-}=\left\{a \in R_{+} \mid w^{-1}(\alpha)>0\right\} \text { and } R_{W}^{+}=\left\{a \in R_{+} \mid w^{-1}(\alpha)<0\right\} .
$$

Let $\gamma_{1}, \ldots, \gamma_{s} \in R_{W}^{-}$and $\gamma_{s+1}, \ldots, \gamma_{k} \in R_{W}^{+}$. 
Recall that

$$
\rho-w(\rho)=\sum_{\beta \in R_{W}^{-}} \beta .
$$

Since $E=E^{*}$, the weight $-\mu$ is a weight of $E$; but then

$$
\begin{aligned}
& w^{-1}\left(\gamma_{1}+\ldots+\gamma_{k}\right)= \\
& \left(w^{-1}\left(\gamma_{1}\right)+\ldots+w^{-1}\left(\gamma_{s}\right)\right)+\left(w^{-1}\left(\gamma_{s+1}\right)+\ldots+w^{-1}\left(\gamma_{k}\right)\right)= \\
& \left(-\lambda-w^{-1}(\mu)\right)+w^{-1}\left(\sum_{\beta \in R_{W}^{-}} \beta\right) .
\end{aligned}
$$

Since $-w^{-1}(\mu)$ is a weight, $\lambda+w^{-1}(\mu) \in Q_{+}(R)$. Hence,

$$
-\left(\lambda+w^{-1}(\mu)\right)+w^{-1}\left(\sum_{\beta \in R_{W}^{-}} \beta\right) \in-Q_{+}(R) .
$$

On the other hand,

$$
w^{-1}\left(\gamma_{s+1}\right)+\ldots+w^{-1}\left(\gamma_{k}\right) \in Q_{+}(R)
$$

and all the $w^{-1}\left(\gamma_{i}\right)$ for $i \leq s$ enter, as summands, $\sum_{\beta \in R_{W}^{-}} w^{-1} \beta$, and therefore cancel each other.

We finally get:

$$
\begin{aligned}
Q_{+}(R) \ni w^{-1}\left(\gamma_{s+1}\right)+\ldots+w^{-1}\left(\gamma_{k}\right) & = \\
& -\left(\lambda+w^{-1}(\mu)\right)+w^{-1}\left(\sum_{\beta \in R_{W}^{-} \backslash\left\{\gamma_{1}, \ldots, \gamma_{s}\right\}} \beta\right) \in-Q_{+}(R) .
\end{aligned}
$$

Thus, $s=k, \lambda=-w^{-1}(\mu)$. In other words,

$$
-\mu=w(\lambda)=-\sum a_{i} \alpha_{i} \text { for } a_{i} \geq 0 .
$$

Since $\lambda$ is a dominant weight, $\lambda=\sum m_{i} \alpha_{i}$, where all the $m_{i} \in \mathbb{Q}$ are positive. This is true for any fundamental weight, as follows from the tables from $[\mathrm{Bu}]$.

By the hypothesis, $l(w)<\mathrm{rk} \mathfrak{g}$, and therefore

$$
w=s_{\alpha_{1}} \ldots s_{\alpha_{r}} \text {, where } r<\operatorname{rkg} \mathfrak{g}
$$

Set

$$
B_{w}:=\left\{\alpha_{i_{1}}, \ldots, \alpha_{i_{r}}\right\} \subset B .
$$

For any $x \in Q(B) \otimes_{\mathbb{Z}} \mathbb{R}$, we have $x-w(x)=-\sum_{\alpha \in B_{w}} n_{\alpha} \alpha$. But then (recall that $\varpi_{j}$ is the $j$ th fundamental weight, see $[\mathrm{Bu}]$ )

$$
\left(x-w(x), \varpi_{j}\right)=0 \text { for some } j \leq \mathrm{rk} \mathfrak{g} .
$$

Therefore, $\left(\lambda, \varpi_{j}\right)=\left(w(\lambda), \varpi_{j}\right)$. Now notice that

$$
\left(\lambda, \varpi_{j}\right)=m_{j}\left(\alpha_{j}, \varpi_{j}\right)>0, \quad\left(w(\lambda), \varpi_{j}\right)=-a_{j}\left(\alpha_{j}, \varpi_{j}\right) \leq 0 .
$$

This contradiction shows that $\varphi_{i}=0$ for $i<\mathrm{rk} \mathfrak{g}$. This, in turn, means that, for $i=$ $1, \ldots, \mathrm{rkg}-1$, there exist short exact sequences of l-homomorphisms

$$
0 \longrightarrow H^{i-1}\left(\mathfrak{g}_{-} ; E / V\right) \longrightarrow H^{i}\left(\mathfrak{g}_{-} ; V\right) \longrightarrow H^{i}\left(\mathfrak{g}_{-} ; E\right) \longrightarrow 0
$$

4.4. Theorem. $H^{2}\left(\mathfrak{g}_{-} ; \mathfrak{g}_{-} \oplus \mathfrak{l}^{(1)}\right)=H^{2}\left(\mathfrak{g}_{-} ; \mathfrak{g}\right) \oplus H^{1}\left(\mathfrak{g}_{-} ;\left(\mathfrak{g}_{-} \oplus \mathfrak{z}\right)^{*}\right)$. 
Proof. Set $E=\mathfrak{g}, V=\mathfrak{g}_{-} \oplus \mathfrak{l}^{(1)}$. By Lemma 4.3,

$$
H^{2}\left(\mathfrak{g}_{-} ; \mathfrak{g}_{-} \oplus \mathfrak{l}^{(1)}\right)=H^{2}\left(\mathfrak{g}_{-} ; \mathfrak{g}\right) \oplus H^{1}\left(\mathfrak{g}_{-} ; \mathfrak{g} /\left(\mathfrak{g}_{-} \oplus \mathfrak{l}^{(1)}\right)\right)
$$

It remains to verify that $\mathfrak{g} /\left(\mathfrak{g}_{-} \oplus \mathfrak{l}^{(1)}\right)=\left(\mathfrak{g}_{-} \oplus \mathfrak{z}\right)^{*}$. Indeed, the Killing form $K$ establishes an isomorphism $\mathfrak{g}=\mathfrak{g}^{*}$, and therefore

$$
\left(\mathfrak{g} /\left(\mathfrak{g}_{-} \oplus \mathfrak{l}^{(1)}\right)\right)^{*}=\left\{x \in \mathfrak{g} \mid K\left(x, \mathfrak{g}_{-} \oplus \mathfrak{l}^{(1)}\right)=0\right\}=\mathfrak{g}_{-} \oplus \mathfrak{z}
$$

where $\mathfrak{z}=\left\{z \in \mathfrak{l} \mid K\left(z, h_{\alpha}\right)=0\right.$ for any $\left.\alpha \in B_{I}\right\}$, as required.

Observe that $\operatorname{dim} \mathfrak{z}$ is equal to the cardinality of $I$, it is 1 in $\S \S 5,7$ and 2 in $\S 6$.

Corollary. Let $B_{1}=B \backslash B_{I}$; let $R_{1}$ be the root system generated by $B_{1}$ and

$$
\mathfrak{g}_{-}^{a b}=\left(\mathfrak{g}_{-} / \mathfrak{g}_{-}^{(1)}\right)^{*}=H^{1}\left(\mathfrak{g}_{-}\right)
$$

1) The following sequence is exact:

$$
\begin{aligned}
& 0 \longrightarrow \mathfrak{g}_{-}^{a b} \longrightarrow \mathfrak{z}^{*} \otimes \mathfrak{g}_{-}^{a b} \longrightarrow H^{1}\left(\mathfrak{g}_{-} ; \mathfrak{g} /\left(\mathfrak{g}_{-} \oplus \mathfrak{l}^{(1)}\right)\right) \longrightarrow H^{1}\left(\mathfrak{g}_{-} ; \mathfrak{g}_{-}^{*}\right) \longrightarrow \\
& H^{2}\left(\mathfrak{g}_{-}\right) \oplus \underset{w \in W\left(R_{1}\right)_{(2)}}{\bigoplus} L^{\rho-w(\rho)} \longrightarrow 0
\end{aligned}
$$

2) If $\operatorname{dim} \mathfrak{z}=1$, then the sequence

$$
0 \longrightarrow H^{1}\left(\mathfrak{g}_{-} ;\left(\mathfrak{g}_{-} \oplus \mathfrak{z}\right)^{*}\right) \longrightarrow H^{1}\left(\mathfrak{g}_{-} ; \mathfrak{g}_{-}^{*}\right) \longrightarrow H^{2}\left(\mathfrak{g}_{-}\right) \longrightarrow 0
$$

is exact. In particular, if $\mathfrak{g}_{-}$is a Heisenberg algebra (the case of contact grading), then

$$
H^{2}\left(\mathfrak{g}_{-} ; \mathfrak{g}_{-} \oplus \mathfrak{l}^{(1)}\right) \simeq H^{2}\left(\mathfrak{g}_{-} ; \mathfrak{g}\right) \oplus S^{2}\left(\mathfrak{g}_{-} / \mathfrak{z}\left(\mathfrak{g}_{-}\right)\right)^{*}=H^{2}\left(\mathfrak{g}_{-} ; \mathfrak{g}\right) \oplus S^{2}\left(\mathfrak{g}_{-1}^{*}\right) .
$$

3) if $\mathfrak{g}_{-}=\mathfrak{g}_{-1}$ (is abelian), then

$$
H^{2}\left(\mathfrak{g}_{-} ; \mathfrak{g}_{-} \oplus \mathfrak{l}^{(1)}\right) \simeq H^{2}\left(\mathfrak{g}_{-} ; \mathfrak{g}\right) \oplus S^{2}\left(\mathfrak{g}_{-}^{*}\right)=H^{2}\left(\mathfrak{g}_{-} ; \mathfrak{g}\right) \oplus S^{2}\left(\mathfrak{g}_{-1}^{*}\right)
$$

Proof. From the short exact sequence

$$
\left.0 \longrightarrow\left(\mathfrak{g}_{-} \oplus \mathfrak{l}\right) /\left(\mathfrak{g}_{-} \oplus \mathfrak{l}^{(1)}\right) \longrightarrow \mathfrak{g} /\left(\mathfrak{g}_{-} \oplus \mathfrak{l}^{(1)}\right)\right) \longrightarrow \mathfrak{g} /\left(\mathfrak{g}_{-} \oplus \mathfrak{l}\right) \longrightarrow 0
$$

where $\mathfrak{g} /\left(\mathfrak{g}_{-} \oplus \mathfrak{l}\right)=\mathfrak{g}_{-}^{*}$ and $\left(\mathfrak{g}_{-} \oplus \mathfrak{l}\right) /\left(\mathfrak{g}_{-} \oplus \mathfrak{l}^{(1)}\right)=\mathfrak{z}^{*}$, we get the long exact sequence

$$
\begin{aligned}
& 0 \longrightarrow \mathfrak{g}_{-}^{a b} \longrightarrow \mathfrak{z}^{*} \otimes H^{1}\left(\mathfrak{g}_{-}\right) \longrightarrow H^{1}\left(\mathfrak{g}_{-} ; \mathfrak{g} /\left(\mathfrak{g}_{-} \oplus \mathfrak{l}^{(1)}\right)\right) \longrightarrow H^{1}\left(\mathfrak{g}_{-} ; \mathfrak{g}_{-}^{*}\right) \stackrel{\psi}{\longrightarrow} \\
& \left.\longrightarrow H^{2}\left(\mathfrak{g}_{-} ; \mathfrak{z}^{*}\right)\left(=H^{2}\left(\mathfrak{g}_{-}\right) \otimes \mathfrak{z}^{*}\right)\right) \longrightarrow \ldots
\end{aligned}
$$

To compute the image of the map $\psi$, consider the $\mathfrak{l}$-module

$$
\begin{aligned}
M= & \left\{f \in \operatorname{Hom}\left(\mathfrak{g}_{-}, \mathfrak{g} /\left(\mathfrak{g}_{-} \oplus \mathfrak{l}^{(1)}\right)\right) \mid X f(Y)-Y f(X)-f([X, Y]) \in \mathfrak{z}^{*}\right. \\
& \text { for any } \left.X, Y \in \mathfrak{g}_{-}\right\} .
\end{aligned}
$$

From the general properties of the long exact sequences $([\underline{F F}])$ we deduce that

$$
\operatorname{Im} \psi=M / Z^{1}\left(\mathfrak{g}_{-} ; \mathfrak{g} /\left(\mathfrak{g}_{-} \oplus \mathfrak{l}^{(1)}\right)\right)
$$

By the BWB theorem,

$$
H^{2}\left(\mathfrak{g}_{-}\right)=\underset{\left\{w \mid l(w)=2, w\left(B \backslash B_{I}\right)>0\right\}}{\oplus} L^{w(\rho)-\rho} .
$$

Since $M$ is the direct sum of its weight subspaces relative to $\mathfrak{h}$, let us study the subspaces $M_{\rho-w(\rho)}$. The weight of $f \in M$ is equal to $w(\rho)-\rho$ if and only if $f$ sends $\mathfrak{n}_{-\gamma}$ to

$$
\left(\mathfrak{g} /\left(\mathfrak{g}_{-} \oplus \mathfrak{l}^{(1)}\right)\right)_{\rho-w(\rho)-\gamma} .
$$

Therefore, either $\rho-w(\rho)-\gamma=\gamma^{\prime} \in R$ or $\rho-w(\rho)-\gamma=0$. 
The second option is ruled out since $\rho-w(\rho)$ is not a root: indeed, $l(w)=2 \neq 1$.

In the first case, $\rho-w(\rho)=\gamma+\gamma^{\prime}$, where $\gamma, \gamma^{\prime} \in R_{+}$. If $\gamma=\gamma^{\prime}$, then $\rho-w(\rho)=2 \gamma$, implying $w^{-1}(\gamma)<0$. But card $\left(R_{W}^{-}\right)=2$ and, $\rho-w(\rho)=2 \gamma=\gamma_{1}+\gamma_{2}$, where $\gamma_{1}, \gamma_{2} \in R_{W}^{-}$. Since one of the $\gamma_{i}$ is equal to $\gamma$, so is the other one. This contradicts the hypothesis: $\gamma_{1} \neq \gamma_{2}$.

Thus, $\rho-w(\rho)=\gamma+\gamma^{\prime}$, where $\gamma \neq \gamma^{\prime}$. It is not difficult to deduce from this that $w^{-1}(\gamma)<0$ and $w^{-1}\left(\gamma^{\prime}\right)<0$. Hence, $f\left(\mathfrak{n}_{-\delta}\right) \neq 0$ only if $w^{-1}(\delta)<0$ for $f \in M_{\rho-w(\rho)}$. In this case $f\left(\mathfrak{n}_{-\delta}\right) \in\left(\mathfrak{g} /\left(\mathfrak{g}_{-} \oplus \mathfrak{l}^{(1)}\right)\right)_{\delta^{\prime}}$, where $w^{-1}\left(\delta^{\prime}\right)<0$. Besides, since $\delta, \delta^{\prime} \notin R$, we have $\left[X_{-\delta}, X_{-\delta^{\prime}}\right]=0$ for the root vectors $X_{-\delta}, X_{-\delta^{\prime}}$. (Indeed, otherwise $\delta, \delta^{\prime} \in R_{W}^{-}$and $l(w)>2$.)

On the other hand, any map $f_{w}: \mathfrak{n}_{-\delta} \longrightarrow \mathfrak{g} /\left(\mathfrak{g}_{-} \oplus \mathfrak{l}^{(1)}\right)$ which vanishes outside the subspace $\mathfrak{n}_{-\gamma} \oplus \mathfrak{n}_{-\gamma^{\prime}}$, where $\left\{\gamma, \gamma^{\prime}\right\}=R_{W}^{-}$, and such that the weight of $f_{w}\left(\mathfrak{n}_{-\gamma}\right)$ is equal to $\gamma^{\prime}$ whereas that of $f_{w}\left(\mathfrak{n}_{-\gamma^{\prime}}\right)$ is $\gamma$ belongs, clearly, to $M_{\rho-w(\rho)}$. This means that $\operatorname{dim} M_{\rho-w(\rho)} \leq 2$.

Let $w=s_{\alpha_{1}} s_{\alpha_{2}}$, where $\alpha_{2} \in B_{1}$ and $\left(\alpha_{1}, \alpha_{2}\right) \neq 0$. Then $\operatorname{dim} M_{\rho-w(\rho)}=1$, as the following calculation shows: let

$$
f\left(X_{-\alpha_{2}}\right)=c X_{\alpha_{1}+\alpha_{2}}^{\prime}, \quad f\left(X_{-\left(\alpha_{1}+\alpha_{2}\right)}\right)=c^{\prime} X_{\alpha_{2}}^{\prime},
$$

where the primed vectors belong to the quotient space. Then, for an appropriate linear constraint on $c$ and $c^{\prime}$, we have

$$
\begin{aligned}
X_{-\left(\alpha_{1}+\alpha_{2}\right)} f\left(X_{-\alpha_{2}}\right)-X_{-\alpha_{2}} f\left(X_{-\left(\alpha_{1}+\alpha_{2}\right)}^{\prime}\right) & = \\
& -c H_{\alpha_{1}+\alpha_{2}}^{\prime}+c^{\prime} H_{\alpha_{2}}^{\prime} \in \mathbb{C} H_{\alpha_{1}}^{\prime} \subset\left(\mathfrak{g}_{-} \oplus \mathfrak{l}\right) /\left(\mathfrak{g}_{-} \oplus \mathfrak{l}^{(1)}\right) .
\end{aligned}
$$

If $w=s_{\alpha_{1}} s_{\alpha_{2}}$, where $\alpha_{1}, \alpha_{2} \in B_{I}$, then $\operatorname{dim} M_{\rho-w(\rho)}=2$. Since, for any such $w$, we have

$$
M_{\rho-w(\rho)} \cap Z^{2}\left(\mathfrak{g}_{-} ; \mathfrak{g} /\left(\mathfrak{g}_{-} \oplus \mathfrak{l}^{(1)}\right)\right) \neq 0,
$$

we get the desired heading 1) of the corollary.

The general statement of heading 2) is straightforward; its particular case a) follows from evident remarks: $H^{1}\left(\mathfrak{g}_{-} ; \mathfrak{g}_{-}^{*}\right)=\mathfrak{g}_{-}^{*} \otimes \mathfrak{g}_{-}^{*}$ and $H^{2}\left(\mathfrak{g}_{-}\right)=E^{2}\left(\mathfrak{g}_{-}^{*}\right)$.

Proof of heading $2 b$ ). Set $\mathfrak{g}_{-}^{\prime}=\mathfrak{g}_{-} / \mathfrak{z}\left(\mathfrak{g}_{-}\right)$. Clearly, $\mathfrak{g}_{-}^{\prime}$ is a trivial $\mathfrak{g}_{-}^{\prime}$-module. Therefore, the sequence

$$
0 \longrightarrow \mathfrak{g}_{-}^{\prime} \longrightarrow \mathfrak{g}_{-}^{\prime} \longrightarrow \mathbb{C} \longrightarrow 0
$$

is exact, hence, so is the sequence

$$
0 \longrightarrow \mathbb{C} \longrightarrow \mathfrak{g}_{-}^{\prime} \otimes H^{1}\left(\mathfrak{g}_{-}\right) \longrightarrow H^{1}\left(\mathfrak{g}_{-} ; \mathfrak{g}_{-}^{\prime}\right) \stackrel{f}{\longrightarrow} H^{2}\left(\mathfrak{g}_{-}\right) \longrightarrow \ldots
$$

It is not difficult to notice that the image of any cocycle $\mathfrak{g}_{-} \longrightarrow \mathfrak{g}_{-}^{\prime}$ under $f$ belongs to $E^{2}\left(\mathfrak{g}_{-}^{\prime}\right)$, hence, $f$ is zero. But then $H^{1}\left(\mathfrak{g}_{-} ; \mathfrak{g}_{-}^{\prime}\right)$ is isomorphic to the subspace $\left(\mathfrak{g}_{-}^{\prime} \otimes \mathfrak{g}_{-}^{\prime}\right)_{0}$ of traceless operators in $\mathfrak{g}_{-}^{\prime} \otimes \mathfrak{g}_{-}^{\prime}$. It remains to notice that $H^{2}\left(\mathfrak{g}_{-}\right) \oplus \mathbb{C}=E^{2}\left(\mathfrak{g}_{-}^{\prime}\right)$.

4.5. The number of $\mathfrak{g}_{0}$-modules. The following Theorem helps to verify the result. Let IR be the number of irreducible components in the $\mathfrak{g}_{0}$-module $H^{2}\left(\mathfrak{g}_{-} ;\left(\mathfrak{g}_{-}, \mathfrak{g}_{0}\right)_{*}\right)$.

Theorem. $I R=\frac{1}{2} c(c+1)+\sum c_{i}$.

Proof. Since $I R=$ card $W(I)_{2}$, let us list the length 2 elements of $W(I)$. Clearly,

$$
W\left(R_{I}\right)_{2}=\left\{w \in W\left(R_{I}\right) \mid l(w)=2\right\} \subset W(I)_{2} .
$$

If $s_{i} s_{j} \in W(I)_{2} \backslash W\left(R_{I}\right)_{2}$, then $s_{j} \in B_{I}$ and $s_{i} \notin B_{I}$. (Indeed, if $s_{j} \notin B_{I}$, then $s_{i} s_{j}\left(a_{j}\right)=$ $-s_{i}\left(a_{j}\right)<0$ which is false. $)$

Furthermore, $\left(\alpha_{i}, \alpha_{j}\right) \neq 0$ since otherwise $s_{i} s_{j}\left(\alpha_{i}\right)=-\alpha_{i}<0$. 
Let rk $R=r$. Let $R=R_{1} \coprod \ldots \coprod R_{s}$, be the representation as the union of connected components. Let $B=\left\{\alpha_{1}, \ldots, \alpha_{r}\right\}$ be a base of $R$. Then

$$
\begin{aligned}
& W(R)_{2}=\left\{s_{i} s_{j} \text { for } i \neq j\right\}= \\
& \left\{s_{i} s_{j} \text { for } i<j\right\} \coprod\left\{s_{i} s_{j} \text { for } i>j \mid\left(\alpha_{i}, \alpha_{j}\right) \neq 0\right\} .
\end{aligned}
$$

It follows that (here edges are counted ignoring multiplicities)

$$
\begin{aligned}
\text { card } W(R)_{2} & =\frac{c(c-1)}{2}+\text { card }\{\text { edges of the Dynkin graph of } R\} \\
& =\frac{c(c-1)}{2}+c-s .
\end{aligned}
$$

If $\alpha \in R \backslash R_{I}$ and $\left(\alpha, R_{I}^{(i)}\right) \neq 0$ for some $i$, then there exists a unique $\beta \in R_{I}^{(i)}$ such that $(\alpha, \beta) \neq 0$. Indeed, if there are two such roots, say, $\beta_{1}$ and $\beta_{2}$, then the Dynkin graph contains a cycle

$$
\alpha-\beta_{1}-\gamma_{1}-\gamma_{2}-\ldots-\gamma_{s}-\beta_{2}-\alpha-
$$

Indeed: $D_{I}^{(i)}$ is connected and $\alpha \notin R_{I}^{(i)}$, hence, $\gamma_{1}, \ldots, \gamma_{s} \in D_{I}^{(i)}$.

All this demonstrates that

$$
\begin{aligned}
\operatorname{card}\left(W_{I}\right)_{2} & =\operatorname{card}\left(W\left(R_{I}\right)\right)_{2}+\sum c_{i}+1 \\
& =\frac{1}{2} c(c-1)+c-s+\sum c_{i}+s=\frac{1}{2} c(c-1)+\sum c_{i} .
\end{aligned}
$$

$\S 5$. The Explicit Results: The Simplest Flags

We consider the standard numbering of vertices of the Dynkin graphs (same as in [OV] or $\mathrm{Bu}$ ).

Let $k_{i}$ be the coefficient of the $i$ th simple root in the expansion of the maximal root with respect to the simple roots. For the nilpotent algebra $\mathfrak{g}_{-}=\underset{-d \leq i \leq-1}{\bigoplus} \mathfrak{g}_{i}$ opposite the maximal parabolic subalgebra $\mathfrak{p}_{i}$ (with the $i$ th selected simple coroot), we have $d=k_{i}$. The vertices labelled by a $k_{i}=1$ correspond to the Hermitian symmetric cases already considered in Go. for the conformal case and in [LPS] for the Riemannian case. For the $X=G / P$, we give the lowest weights of the (nonholonomic only for $k_{i} \neq 1$ ) N-Weyl tensors with respect to the Levi subgroup corresponding to the $\mathbb{Z}$-grading of $\mathfrak{g}$ for which the selected coroot (vertex) is of degree -1 , the other coroots being of degree 0 .

The weights of the simple roots are given by the columns of the Cartan matrix. Set $H^{1}:=H^{1}\left(\mathfrak{g}_{-} ;\left(\mathfrak{g}_{-} \oplus \mathfrak{z}\right)^{*}\right)$ and $H^{2}:=H^{2}\left(\mathfrak{g}_{-} ; \mathfrak{g}\right)$.

Theorem. Tables $1-4$ give the lowest weights of irreducible $\mathfrak{l}$-components in $H^{2}$ in terms of the Cartan matrix (CM): they are easier to compute, and in terms of the fundamental weights $(F W)$ : they are more conventional. ${ }^{4}$

The highest weights of irreducible $\mathfrak{l}$-components in $H^{1}$ are given in terms of the fundamental weights only. To save space, the column $H^{1}$ contains all the irreducible l-modules we have found but one: for each algebra, for the ith node, there always is a component with the highest weight $(0, \ldots, 0,2,0, \ldots, 0)$ with the 2 on the ith place.

We give the weights of the $\widehat{\mathfrak{g}_{0}}=\mathfrak{l}^{(1)}$-modules that constitute non-conformal part of the NRiemannian tensor with respect to the same Cartan subalgebra of $\mathfrak{g}_{0}=\mathfrak{l}$ we used to describe N-Weyl tensors.

\footnotetext{
${ }^{4}$ SuperLie converts FW-weights to CM-weights or the other way round in seconds.
} 
The order of N-Riemannian tensors coincides with the number occupying the place whose number is equal to the number of the node. Therefore orders of all structure functions are equal to 2, except one cases of order 4 for $\mathfrak{g}(2)$ and several cases of order 3 for $\mathfrak{f}(4)$ and $\mathfrak{o}(2 n+1)$.

Remark. The positive coordinates of lowest weights are not mistakes: when expressed in terms of the Cartan matrix (CM) they only occur at the place governed by the center of $\mathfrak{l}$; the remaining (non-positive) coordinates correspond to the semi-simple part of $\mathfrak{l}$.

\section{§6. The explicit Results: Case 3) of Yamaguchi's theorem}

Theorem. In the cases of heading 3) of Yamaguchi's theorem, (for completeness we consider the last "selected" simple coroot of $\mathfrak{s l}$ as well) the lowest weights of the $\mathfrak{g}_{0}$-module $H^{2}\left(\mathfrak{g}_{-} ; \mathfrak{g}\right)$ and highest weights of $H^{1}\left(\mathfrak{g}_{-} ;\left(\mathfrak{g}_{-} \oplus \mathfrak{z}\right)^{*}\right)$ and their degrees are listed below in this section under Tables $1-4$. When the cocycle is simple-looking it is also given.

a) $\mathfrak{g}=\mathfrak{s l}(n+1)$ : (the weights are given with respect to the standard generators of the Cartan subalgebra of $\mathfrak{g}$, i.e., $\left.e_{1}^{1}-e_{2}^{2}, e_{2}^{2}-e_{3}^{3}, \ldots, e_{n}^{n}-e_{n+1}^{n+1}\right)$

Selected simple coroots: $(1,2)$

1) $\operatorname{deg}=0$ of weight $(-3,3,0,-1,0, \ldots, 0,-1)$;

2) $\operatorname{deg}=2$ of weight $(4,-1,-1,0, \ldots, 0,-1)$;

$3) \operatorname{deg}=3$ of weight $(0,4,-3,0, \ldots, 0,-1)$.

Selected simple coroots: $(1, i)$, where $3 \leq i \leq n-2$. To make the answer graphic, the weight is represented as the sum of a constant part and a part that depends on $i$ (the $i$ th place being $-3,-3$ and 2 , respectively; this variable summand only appears three times):

1) $\operatorname{deg}=0$ of weight $(-1,0, \ldots, 0,-1)+(\ldots, 0,-1,0,-3,-2,0, \ldots)$;

2) $\operatorname{deg}=0$ of weight $(-1,0, \ldots, 0,-1)+(\ldots, 0,-2,-3,0,-1,0, \ldots)$;

3) $\operatorname{deg}=1$ of weight $(3,-2,0, \ldots, 0,-1)+(\ldots, 0,-1,2,-1,0, \ldots)$;

4) $\operatorname{deg}=1$ of weight $(4,-1,-1,0,0,0,-1)$.

Selected simple coroots: $(1, n-1)$ (one weight differs from the above)

1) $\operatorname{deg}=0$ of weight $(-1,0, \ldots, 0,-1,0,3,-3)$ (as above);

2) $\operatorname{deg}=1$ of weight $(-1,0, \ldots, 0,-3,4,0)$;

$3) \operatorname{deg}=1$ of weight $(3,-2,0, \ldots, 0,-1,2,-2)$ (as above);

$4) \operatorname{deg}=1$ of weight $(4,-1,-1,0, \ldots, 0,-1)$ (as above).

Exceptional cases:

sl(5) Selected simple coroots: $(1,2)$

1) $\operatorname{deg}=0$ of weight $(-3,3,0,-2)$;

2) $\operatorname{deg}=2$ of weight $(4,-1,-1,-1)$;

$3) \operatorname{deg}=3$ of weight $(0,4,-3,-1)$.

$\mathfrak{s l}(5)$ Selected simple coroots: $(1,3)$

1) $\overline{\mathrm{deg}}=0$ of weight $(-2,0,3,-3)$;

2) $\operatorname{deg}=1$ of weight $(-1,-3,4,0)$;

3) $\operatorname{deg}=1$ of weight $(3,-3,2,-2)$;

4) $\operatorname{deg}=1$ of weight $(4,-1,-1,-1)$.

$\mathfrak{s l}(4)$ Selected simple coroots: $(1,2)$

1) $\operatorname{deg}=1$ of weight $(-4,4,0)$;

2) $\operatorname{deg}=2$ of weight $(4,-1,-2)$;

$3) \operatorname{deg}=3$ of weight $(0,4,-4)$. 
$\underline{\mathfrak{s l}(4)}$ Selected simple coroots: $(1, n)$ :

\begin{tabular}{|c|c|c|c|}
\hline degree & vector: $n>4$ & weight: $n>4$ & weight: $n=4$ \\
\hline 1 & $\left(e_{2}^{n+1}\right)^{*} d e_{1}^{2} d e_{1}^{3}$ & $(4,-1,-1,0, \ldots, 0,-1)$ & $(4,-1,-2)$ \\
\hline 1 & symmetric to the above under & symmetric & symmetric \\
& $n+1 \longleftrightarrow 1, n \longleftrightarrow 2$, etc. & to the above & to the above \\
\hline 2 & $\left(e_{2}^{n}\right)^{*} d e_{1}^{2} d e_{n}^{n+1}$ & $(3,-2,0, \ldots, 0,-2,3)$ & $(3,-4,3)$ \\
\hline
\end{tabular}

The exceptional case $\mathfrak{s l}(3)$ :

1) $\operatorname{deg}=4:\left(e_{3}^{2}\right)^{*} d\left(e_{2}^{3}\right) d\left(e_{1}^{3}\right)$ of weight $(-1,5)$;

2) symmetric to the above.

$\underline{H^{1}}$ : all vectors except (3) exist for $2 \leq i \leq n-1$

(1) $\operatorname{deg}=1:\left((i-1) e_{1}^{1}-e_{2}^{2}-\ldots-e_{i}^{i}\right)^{*} d e_{i}^{i+1}$ of weight $(\ldots, 0,-1,2,-1, .0, \ldots)$;

(2) $\operatorname{deg}=1:\left((i-1) e_{1}^{1}-e_{2}^{2}-\ldots-e_{i}^{i}\right)^{*} d e_{1}^{2}$ of weight $(2,-1,0, \ldots)$;

(3) $\operatorname{deg}=2:\left(e_{i}^{i+1}\right)^{*} d e_{i-1}^{i+2}-\left(e_{i-1}^{i+1}\right)^{*} d e_{i}^{i+2}-\left(e_{i}^{i+2}\right)^{*} d e_{i-1}^{i+1}+\left(e_{i-1}^{i+2}\right)^{*} d e_{i}^{i+1}$ of weight

$(\ldots, 0,-1,0,2,0,-1,0, \ldots)$ (for $i=n-1$ of weight $(\ldots, 0,-1,0,2,0)$ ), exists for $3 \leq i \leq n-1$;

(4) $\operatorname{deg}=2:\left(e_{i}^{i+1}\right)^{*} d e_{i}^{i+1}$ of weight $(\ldots, 0,-2,4,-2,0, \ldots)$;

(5) $\operatorname{deg}=2$ : of weight $(1,0, \ldots, 0,1,-1,0, \ldots)$ :

$$
\begin{aligned}
& (n+1)\left((i-1) e_{1}^{1}-e_{2}^{2}-\ldots-e_{i}^{i}\right)^{*} d e_{1}^{i+1}- \\
& (i-1)(n-i+1) \sum_{2 \leq j \leq i}\left(\left(e_{j}^{i+1}\right)^{*} d e_{1}^{j}\right)+(n+2-i) \sum_{2 \leq j \leq i}\left(\left(e_{1}^{j}\right)^{*} d e_{j}^{i+1}\right) ;
\end{aligned}
$$

(6) $\operatorname{deg}=2:\left(e_{1}^{2}\right)^{*} d e_{1}^{2}$ of weight $(4,-2,0, \ldots)$.

b) $\mathfrak{s p}(2 n)$ :

\begin{tabular}{|c|c|c|c|}
\hline degree & weight: $n>3$ & weight: $n=3$ & $H^{1}$ \\
\hline-1 & $(-2,0, \ldots, 0,-1,-2,3)$ & $(-3,-2,3)$ & - \\
\hline 1 & $(4,-3,0, \ldots, 0,-2,2)$ & $(4,-5,2)$ & $(0, \ldots, 0,-2,2)$ \\
1 & $(5,-2,-1,0, \ldots, 0)$ & $(5,-2,-1)$ & $(2,-1,0, \ldots, 0)$ \\
\hline
\end{tabular}

There are also irreducible components in $H^{1}$ of degree 2 and their weights are

$$
(0, \ldots, 0,-2,0,2), \quad(0, \ldots, 0,-4,4), \quad(1,0, \ldots, 0,-1,1), \quad(4,-2,0, \ldots, 0) .
$$

The exceptional case $\mathfrak{s p}(4)$ :

$\underline{H^{2}}: \operatorname{deg}=3$ of weight $(6,-3)$ and $\operatorname{deg}=4$ of weight $(-4,5)$.

$\underline{H^{1}}: \operatorname{deg}=1$ of weights $(-2,2)$ and $(2,-1) \operatorname{deg}=2$ of weights $(-4,4),(0,1)$ and $(4,-2)$

\section{$\S 7$. The EXPlicit Results: the $G(2)$-StruCtures}

7.1. The $G(2)$-structure. Let $M$ be a manifold with the $G=G(2)$-structure, i.e., the $G(2)$-module $T_{m} M$ is isomorphic to the 1 st fundamental module, cf. [B]. We wondered to what extent the "positive 3-form" (an invariant of the $G(2)$-structure similar to the metric in the Riemannian case, i.e., for the $\mathfrak{o}(n)$-structure), see [B], can be reduced to a canonical form. Below is a description of the space of obstructions to canonicity. Since $\mathfrak{g}(2) \subset \mathfrak{o}(7)$ and $\mathfrak{o}(n)_{1}=0$ for $n>2$, it is easy to see that $\left(\mathfrak{g}_{-1}, \mathfrak{g}_{0}\right)_{*}=\mathfrak{g}_{-1} \oplus \mathfrak{g}_{0}$.

Statement. As $\mathfrak{g}_{0}$-module, $H^{2}\left(\mathfrak{g}_{-1} ;\left(\mathfrak{g}_{-1}, \mathfrak{g}_{0}\right)_{*}\right)$ is the direct sum of the irreducible $G(2)$ modules whose highest weights and orders of the corresponding structure function are given 
by the following table:

\begin{tabular}{|c|c|}
\hline weight & order \\
\hline$(0,0),(0,1),(1,0),(2,0)$ & 1 \\
\hline$(0,2)$ & 2 \\
\hline
\end{tabular}

The requirement of vanishing of order 1 structure functions (for the corresponding equations, see $[\mathrm{B}$ ) is an analog of Wess-Zumino constraints in supergravity WB].

From the list of $\mathbb{Z}$-gradings of simple Lie algebras we know that there is no analog of classical domain with the $G=C G(2)$-structure, i.e., conformal, even nonholonomic one. Contrariwise, one and only one of the exceptional Lie superalgebras has such a grading. Let us compute the corresponding space of structure functions.

7.2. The $C G(2)$-structure on $\mathbb{C} P^{1,7}$ with a nonholonomic distribution. We consider $\mathbb{C} P^{1,7}$ as the quotient of the simple Lie supergroup $A G(2)$ modulo the parabolic subalgebra corresponding to the grading $(1,0,0)$ for the Cartan matrix, cf. GL2, where the Lie superalgebra $\mathfrak{a} \mathfrak{g}(2)=\operatorname{Lie}(A G(2))$ is presented:

$$
\left(\begin{array}{ccc}
0 & 1 & 0 \\
-1 & 2 & -3 \\
0 & -1 & 2
\end{array}\right)
$$

Here $\mathfrak{g}_{0}=\mathfrak{g}(2) \oplus \mathfrak{z}$ for a 1-dimensional $\mathfrak{z}$; let $\widehat{\mathfrak{g}_{0}}=\mathfrak{g}(2)$. It is now not as easy as in sec. 7.1 to see that $\left(\mathfrak{g}_{-}, \mathfrak{g}_{0}\right)_{*}=\mathfrak{a} \mathfrak{g}(2)$ and $\left(\mathfrak{g}_{-}, \widehat{\mathfrak{g}_{0}}\right)_{*}=\mathfrak{g}_{-} \oplus \widehat{\mathfrak{g}_{0}}$, but still true. No super version of the BWB theorem exists (cf. [Pe, [PS] ) to help us, so, to obtain the following statement, we used SuperLie.

Statement. As $\mathfrak{g}_{0}=\mathfrak{s l}(2) \oplus \mathfrak{g}(2)$-module, $H^{2}\left(\mathfrak{g}_{-1} ; \mathfrak{a} \mathfrak{g}(2)\right)$ - the space of $N$-conformal structure functions - is an irreducible module with highest weight $(5,0,1)$.

As $\widehat{\mathfrak{g}_{0}}=\mathfrak{z} \oplus \mathfrak{g}(2)$-module, $H^{2}\left(\mathfrak{g}_{-1} ;\left(\mathfrak{g}_{-}, \widehat{\mathfrak{g}_{0}}\right)_{*}\right)$ - the space of $N$-Riemannian structure functions - is the direct sum of irreducible modules whose highest weights are as follows:

$$
(5,0,1), \quad(6,1,0), \quad(7,0,1) .
$$

Remark. In our attempt to understand the meaning of tensors $V\left(\mathfrak{g}_{i}\right)$ in $[\mathrm{B}$, where exterior powers of $\mathfrak{g}_{-1}$ appear, we conjectured that these $V\left(\mathfrak{g}_{i}\right)$ might be related with the $G(2)$ structure on a purely odd superspace. However, having computed the corresponding structure functions

\begin{tabular}{|c|c|}
\hline weight & order \\
\hline$(0,1),(1,0),(3,0)$ & 1 \\
\hline$(0,1)$ & 2 \\
\hline
\end{tabular}

we see that they do not coincide with the $V\left(\mathfrak{g}_{i}\right)$, so the meaning of these $V\left(\mathfrak{g}_{i}\right)$ 's remains a mystery to us. 
Table 1

\begin{tabular}{|c|c|c|c|c|c|}
\hline $\mathfrak{g}$ & Node & $k_{i}$ & $\mathrm{CM}$ & FW & $H^{1}$ \\
\hline \multirow[t]{2}{*}{$\mathfrak{g}(2)$} & 1 & 3 & $(8,-4)$ & $(4,0)$ & $(4,2)$ \\
\hline & 2 & 2 & $(-7,4)$ & $(-2,1)$ & $(2,2)$ \\
\hline \multirow[t]{4}{*}{$\mathfrak{f}(4)$} & 1 & 2 & $(3,0,-1,-1)$ & $(0,-3,-3,-2)$ & $(2,3,2,1)$ \\
\hline & 2 & 4 & $\begin{array}{c}(0,3,-2,-1) \\
(-3,4,-1,-2)\end{array}$ & $\begin{array}{l}(-1,-2,-3,-2) \\
(-2,-1,2,-2)\end{array}$ & $\begin{array}{l}(0,3,2,1) \\
(1,2,1,0)\end{array}$ \\
\hline & 3 & 3 & $\begin{array}{c}(0,-6,4,0) \\
(-1,-2,3,-3)\end{array}$ & $\begin{array}{c}(-2,-4,0,0) \\
(-2,-3,-1,-2)\end{array}$ & $\begin{array}{l}(0,2,2,0) \\
(0,1,2,0)\end{array}$ \\
\hline & 4 & 2 & $(0,-2,-1,4)$ & $(-2,-4,-2,1)$ & $(0,2,2,2)$ \\
\hline \multirow[t]{6}{*}{$\mathfrak{e}(6)$} & 1 & 1 & $(3,0,-1,0,0,-1)$ & $(1,-1,-3,-2,-1,-2)$ & $(2,2,2,1,0,1)$ \\
\hline & 2 & 2 & $\begin{array}{c}(0,3,-2,0,0,-1) \\
(-2,3,0,-1,0,-2)\end{array}$ & $\begin{array}{c}(0,0,-3,-2,-1,-2) \\
(-1,0,-2,-2,-1,-2)\end{array}$ & $\begin{array}{l}(1,2,1,0,0,0) \\
(0,2,2,1,0,1)\end{array}$ \\
\hline & 3 & 3 & $\begin{array}{c}(0,-3,4,-3,0,0) \\
(0,-2,3,0,-1,-3) \\
(-1,0,3,-2,0,-3)\end{array}$ & $\begin{array}{c}(-1,-2,0,-2,-1,0) \\
(-1,-2,-1,-1,-1,-2) \\
(-1,-1,-1,-2,-1,-2)\end{array}$ & $\begin{array}{l}(0,1,2,1,0,0) \\
(0,0,2,1,0,1) \\
(0,1,2,0,0,1)\end{array}$ \\
\hline & 4 & 2 & $\begin{array}{c}(0,0,-2,3,0,-1) \\
(0,-1,0,3,-2,-2)\end{array}$ & $\begin{array}{c}(-1,-2,-3,0,0,-2) \\
(-1,-2,-2,0,-1,-2)\end{array}$ & $\begin{array}{l}(0,0,1,2,1,0) \\
(0,1,2,2,0,1)\end{array}$ \\
\hline & 5 & 1 & $(0,0,-1,0,3,-1)$ & $(-1,-2,-3,-1,1,-2)$ & $(0,1,2,2,2,1)$ \\
\hline & 6 & 2 & $(0,-1,-1,-1,0,4)$ & $(-1,-2,-2,-2,-1,1)$ & $(0,1,2,1,0,2)$ \\
\hline \multirow[t]{7}{*}{$\mathfrak{e}(7)$} & 1 & 1 & $(3,0,-1,0,0,-1,0)$ & $(1,-1,-3,-4,-3,-2,-2)$ & $(2,2,2,2,1,0,1)$ \\
\hline & 2 & 2 & $\begin{array}{c}(0,3,-2,0,0,-1,0) \\
(-2,3,0,-1,0,-1,0)\end{array}$ & $\begin{array}{c}(0,0,-3,-4,-3,-2,-2) \\
(-1,0,-2,-4,-3,-2,-2)\end{array}$ & $\begin{array}{l}(1,2,1,0,0,0,0) \\
(0,2,2,2,1,0,1)\end{array}$ \\
\hline & 3 & 3 & $\begin{array}{c}(0,-2,3,0,-1,-1,-1) \\
(-1,0,3,-2,0,-1,0)\end{array}$ & $\begin{array}{l}(-1,-2,-1,-3,-3,-2,-2) \\
(-1,-1,-1,-4,-3,-2,-2)\end{array}$ & $\begin{array}{l}(0,1,2,1,0,0,0) \\
(0,0,2,2,1,0,1)\end{array}$ \\
\hline & 4 & 4 & $\begin{array}{c}(0,0,-2,3,-2,-1,0) \\
(0,0,-2,3,0,-2,-2) \\
(0,-1,0,3,-2,-1,-2)\end{array}$ & $\begin{array}{l}(-1,-2,-3,-2,-3,-2,-1) \\
(-1,-2,-3,-2,-2,-2,-2) \\
(-1,-2,-2,-2,-3,-2,-2)\end{array}$ & $\begin{array}{l}(0,0,1,2,1,0,0) \\
(0,0,0,2,1,0,1) \\
(0,0,1,2,0,0,1)\end{array}$ \\
\hline & 5 & 3 & $\begin{array}{c}(0,0,0,-3,4,0,0) \\
(0,0,-1,0,3,-3,-1)\end{array}$ & $\begin{array}{c}(-1,-2,-3,-4,0,0,-2) \\
(-1,-2,-3,-3,-1,-2,-2)\end{array}$ & $\begin{array}{l}(0,0,0,1,2,1,0) \\
(0,0,1,2,2,0,1)\end{array}$ \\
\hline & 6 & 2 & $(0,0,0,-1,-1,4,0)$ & $(-1,-2,-3,-4,-2,1,-2)$ & $(0,0,1,2,2,2,1)$ \\
\hline & 7 & 2 & $(0,0,-1,0,-1,-1,3)$ & $(-1,-2,-3,-3,-3,-2,0)$ & $(0,0,1,2,1,0,2)$ \\
\hline \multirow[t]{8}{*}{$\mathfrak{e}(8)$} & 1 & 2 & $(4,-1,-1,0,0,0,0,0)$ & $(-1,-2,-4,-5,-6,-4,-2,-3)$ & $(2,2,2,2,2,1,0,1)$ \\
\hline & 2 & 3 & $\begin{array}{c}(0,4,-3,0,0,0,0,0) \\
(-3,3,0,-1,0,0,0,0) \\
\end{array}$ & $\begin{array}{c}(0,0,-4,-5,-6,-4,-2,-3) \\
(-2,-1,-3,-5,-6,-4,-2,-3)\end{array}$ & $\begin{array}{l}(1,2,1,0,0,0,0,0) \\
(0,2,2,2,2,1,0,1) \\
\end{array}$ \\
\hline & 3 & 4 & $\begin{array}{c}(-1,-2,3,0,-1,0,0,0) \\
(-2,0,3,-2,0,0,0,0)\end{array}$ & $\begin{array}{l}(-2,-3,-2,-4,-6,-4,-2,-3) \\
(-2,-2,-2,-5,-6,-4,-2,-3) \\
\end{array}$ & $\begin{array}{l}(1,2,1,0,0,0,0,0) \\
(0,2,2,2,2,1,0,1) \\
\end{array}$ \\
\hline & 4 & 5 & $\begin{array}{c}(-1,0,-2,3,0,-1,0,-1) \\
(-1,-1,0,3,-2,0,0,0)\end{array}$ & $\begin{array}{l}(-2,-3,-4,-3,-5,-4,-2,-3) \\
(-2,-3,-3,-3,-6,-4,-2,-3) \\
\end{array}$ & $\begin{array}{l}(0,0,1,2,1,0,0,0) \\
(0,0,0,2,2,1,0,1)\end{array}$ \\
\hline & 5 & 6 & $\begin{array}{c}(-1,0,0,-2,3,-2,0,0) \\
(-1,0,0,-2,3,0,-1,-2) \\
(-1,0,-1,0,3,-2,0,-2)\end{array}$ & $\begin{array}{l}(-2,-3,-4,-5,-4,-4,-2,-2) \\
(-2,-3,-4,-5,-4,-3,-2,-3) \\
(-2,-3,-4,-4,-4,-4,-2,-2)\end{array}$ & $\begin{array}{l}(0,0,0,1,2,1,0,0) \\
(0,0,0,0,2,1,0,1) \\
(0,0,0,1,2,0,0,1)\end{array}$ \\
\hline & 6 & 4 & $\begin{array}{c}(-1,0,0,0,-2,3,0,0) \\
(-1,0,0,-1,0,3,-2,-1) \\
\end{array}$ & $\begin{array}{c}(-2,-3,-4,-5,-6,-3,0,-3) \\
(-2,-3,-4,-5,-5,-4,-2,-1)\end{array}$ & $\begin{array}{l}(0,0,0,0,1,2,1,0) \\
(0,0,0,1,2,2,0,1)\end{array}$ \\
\hline & 7 & 2 & $(-1,0,0,0,0,-1,2,0)$ & $(-1,0,0,0,-1,0,3,0)$ & $(0,0,0,1,2,2,2,1)$ \\
\hline & 8 & 3 & $(-1,0,0,0,-1,0,0,2)$ & $(-1,0,0,-1,0,-1,0,3)$ & $(0,0,0,1,2,1,0,2)$ \\
\hline
\end{tabular}


Table $2 \mathfrak{o}(2 n)$ :

\begin{tabular}{|c|c|c|c|c|}
\hline Node & $k(i)$ & $r k$ & weight & $H^{1}$ \\
\hline \multirow[t]{2}{*}{1} & \multirow[t]{2}{*}{1} & 4 & $(2,0,-1,-1)$ & $(2211)$ \\
\hline & & $4+l$ & $(2,0, \underbrace{-2, \ldots,-2}_{l},-1,-1)$ & $(\underbrace{2 \ldots 2}_{l-2}, 11)$ \\
\hline 2 & 2 & $5+l$ & $\begin{array}{c}(0,1,-2, \underbrace{-2, \ldots,-2}_{l},-1,-1) \\
(-1,1,-1, \underbrace{-2, \ldots,-2}_{l},-1,-1)\end{array}$ & $\begin{array}{l}(02211) \\
(12100)\end{array}$ \\
\hline 3 & 2 & $6+l$ & $\begin{array}{l}(-1,-2,0,1, \underbrace{-2, \ldots,-2}_{l},-1,-1) \\
-1,0,1,-2, \underbrace{-2, \ldots,-2}_{l},-1,-1)\end{array}$ & $\begin{array}{l}(0 \underbrace{2 \ldots 2}_{l-3} 11) \\
(121 \underbrace{0 \ldots 0}_{l-3})\end{array}$ \\
\hline $\begin{array}{c}3+k \\
k<r k-6\end{array}$ & 2 & 7 & $\begin{array}{l}(-1, \underbrace{-2, \ldots,-2}_{k}-2,0,-1,-1,-1) \\
(-1, \underbrace{-2, \ldots,-2}_{k},-1,0,-2,-1,-1)\end{array}$ & $\begin{array}{l}(002211) \\
(012100)\end{array}$ \\
\hline & & $>7$ & $\begin{array}{l}(-1, \underbrace{-2, \ldots,-2}_{k}-2,0,-1, \underbrace{-2, \ldots,-2}_{l},-1,-1) \\
(-1, \underbrace{-2, \ldots,-2}_{k},-1,0,-2, \underbrace{-2, \ldots,-2}_{l},-1,-1)\end{array}$ & $\begin{array}{l}(0 \ldots 02211) \\
(0 \ldots 012100)\end{array}$ \\
\hline$\ldots$ & $\ldots$ & $\ldots$ & $\ldots$ & $\ldots$ \\
\hline fork & 2 & 4 & $\begin{array}{l}(0,1,-1,-1) \\
(-1,1,-1,0) \\
(-1,1,0,-1)\end{array}$ & $\begin{array}{l}(0211) \\
(1201) \\
(1210)\end{array}$ \\
\hline & & 5 & $\begin{array}{l}(-1,-2,0,-1,0) \\
(-1,-2,0,0,-1) \\
(-1,0,1,-1,-1)\end{array}$ & $\begin{array}{l}(00211) \\
(01201) \\
(01210)\end{array}$ \\
\hline & & $\begin{array}{c}5+k \\
k<r k-5\end{array}$ & $\begin{array}{l}(-1, \underbrace{-2, \ldots,-2}_{k},-2,0,-1,0) \\
(-1, \underbrace{-2, \ldots,-2}_{k},-2,0,0,-1) \\
(-1, \underbrace{-2, \ldots,-2}_{k},-1,0,-1,-1)\end{array}$ & $\begin{array}{l}(0 \ldots 00211) \\
(0 \ldots 01201) \\
(0 \ldots 01210)\end{array}$ \\
\hline
\end{tabular}


Table $3 \mathfrak{o}(2 n+1)$ :

\begin{tabular}{|c|c|c|c|c|}
\hline Node & $k(i)$ & $r k$ & weight & $H^{1}$ \\
\hline \multirow[t]{3}{*}{1} & \multirow[t]{3}{*}{1} & 2 & $(3,1)$ & $(22)$ \\
\hline & & 3 & $(2,0,-2)$ & $(222)$ \\
\hline & & $3+k$ & $(2,0, \underbrace{-2, \ldots,-2}_{k})$ & $(2 \ldots 2)$ \\
\hline \multirow[t]{2}{*}{2} & \multirow[t]{2}{*}{2} & 3 & $\begin{array}{c}(0,1,-2) \\
(-1,1,-1)\end{array}$ & $\begin{array}{l}(022) \\
(121)\end{array}$ \\
\hline & & $3+k$ & $\begin{array}{c}(0,1,-2, \underbrace{-2, \ldots,-2}_{k}) \\
(-1,1,-1, \underbrace{-2, \ldots,-2}_{k})\end{array}$ & $\begin{array}{c}(02 \ldots 2) \\
(1210 \ldots 0)\end{array}$ \\
\hline 3 & 2 & $4+k$ & $\begin{array}{l}(-1,-2,0,1, \underbrace{-2, \ldots,-2}_{k}) \\
(-1,0,1,-2 \underbrace{-2, \ldots,-2}_{k})\end{array}$ & $\begin{aligned} &(002 \ldots 2) \\
&(01210 \ldots 0)\end{aligned}$ \\
\hline$\ldots$ & $\ldots$ & $\ldots$ & $\ldots$ & $\ldots$ \\
\hline $\begin{array}{l}\text { penul- } \\
\text { timate }\end{array}$ & 2 & $5+k$ & $\begin{array}{l}(-1, \underbrace{-2, \ldots,-2}_{k}-2,0,-1,-2) \\
(-1,-2, \ldots,-2,-1,0,-2,-2)\end{array}$ & $\begin{array}{l}(0 \ldots 022) \\
(0 \ldots 0121)\end{array}$ \\
\hline \multirow[t]{3}{*}{ last } & \multirow[t]{3}{*}{1} & 2 & $(0,3)$ & - \\
\hline & & 3 & $(-1,0,3)$ & (123) \\
\hline & & $3+k$ & $(-1, \underbrace{-2, \ldots,-2}_{k},-1,1)$ & $(0 \ldots 0123)$ \\
\hline
\end{tabular}

Table $4 \mathfrak{s p}(2 n)$ :

\begin{tabular}{|c|c|c|c|c|}
\hline Node & $k(i)$ & $r k$ & weight & $H^{1}$ \\
\hline \multirow[t]{3}{*}{1} & \multirow[t]{3}{*}{2} & 2 & $(3,0)$ & - \\
\hline & & 3 & $(2,1,-1)$ & - \\
\hline & & $3+k$ & $(2,-1, \underbrace{-2, \ldots,-2}_{k},-1)$ & - \\
\hline \multirow[t]{2}{*}{2} & \multirow[t]{2}{*}{2} & 3 & $\begin{array}{l}(1,2,-1) \\
(-2,1,0)\end{array}$ & (121) \\
\hline & & $4+k$ & $\begin{array}{l}(1,2,-2, \underbrace{-2, \ldots,-2}_{k},-1) \\
(-2,0,-1, \underbrace{-2, \ldots,-2}_{k},-1)\end{array}$ & $\begin{array}{l}(1210 \ldots 0) \\
(12 \ldots 21)\end{array}$ \\
\hline .. & $\ldots$ & $\ldots$ & $\ldots$ & $\ldots$ \\
\hline $\begin{array}{l}\text { pen-penul- } \\
\text { timate }\end{array}$ & 2 & $4+k$ & $\begin{array}{l}(\underbrace{-2, \ldots,-2}_{k},-2,0,1,-1) \\
(\underbrace{-2, \ldots,-2}_{k}, 0,1,-2,-1)\end{array}$ & $\begin{array}{l}(0 \ldots 01210) \\
(0 \ldots 01211)\end{array}$ \\
\hline $\begin{array}{l}\text { penul- } \\
\text { timate }\end{array}$ & 2 & $3+k$ & $\begin{array}{c}(\underbrace{-2, \ldots,-2}_{k}-2,1,0) \\
(\underbrace{-2, \ldots,-2}_{k},-1,0,-1)\end{array}$ & $(0 \ldots 0121)$ \\
\hline \multirow[t]{2}{*}{ last } & \multirow[t]{2}{*}{1} & 2 & $(1,3)$ & $(22)$ \\
\hline & & $3+k$ & $(\underbrace{-2, \ldots,-2}_{k},-2,-1,1)$ & $(0 \ldots 022)$ \\
\hline
\end{tabular}




\section{REFERENCES}

[AS] Agrachev A., Sachkov Yu., Control Theory from the Geometric Viewpoint, Springer, 2004, 412 pp.

[AW] Atiyah, M.; Witten, E., $M$-theory dynamics on a manifold of $G_{2}$ holonomy. Adv. Theor. Math. Phys. 6 (2002), no. 1, 1-106 MR1992874 (2004f:53046)

[BGG] Bernstein I. N., Gelfand I. M., Gelfand S. I. Differential operators on the base affine space and a study of $\mathfrak{g}$-modules. In: Lie groups and their representations (Proc. Summer School, Bolyai János Math. Soc., Budapest, 1971). Halsted, New York, 1975, 21-64

[BR] Biswas I., Raina A., Projective structures on a Riemann surface. Internat. Math. Res. Notices, 1996, 753-768; II.Internat. Math. Res. Notices, 1999, no. 13, 685-716; III. Differential Geom. Appl. 15 (2001), no. 3, 203-219.

[Bl] Bloch, A. M. Nonholonomic mechanics and control. With the collaboration of J. Baillieul, P. Crouch and J. Marsden. With scientific input from P. S. Krishnaprasad, R. M. Murray and D. Zenkov. Interdisciplinary Applied Mathematics, 24. Systems and Control. Springer-Verlag, New York, 2003. xx+483 $\mathrm{pp}$

[Bu] Bourbaki N., Lie groups and Lie algebras. Chapters 4-6. Translated from the 1968 French original by Andrew Pressley. Elements of Mathematics (Berlin). Springer-Verlag, Berlin, 2002. xii+300 pp.

[B] Bryant R. L., Some remarks on $G_{2}$-structures, math.DG/0305124

[C1] Čap, A., Schichl, H., Parabolic geometries and canonical Cartan connections. Hokkaido Math. J. 29 (2000), no. 3, 453-505.

[C2] Cap A., Correspondence spaces and twistor spaces for parabolic geometries; arXiv.org: math.DG/0102097; ESI preprint 989

[C3] Cap A., Slovák J., Weyl structures for parabolic geometries. Math. Scand. 93 (2003), no. 1, 53-90. math.DG/0001166 math.DG/0001166

[C4] Cap A., Slovák J., Souček V., Bernstein-Gelfand-Gelfand sequences. Ann. of Math. (2) 154 (2001), no. $1,97-113$.

[C5] Čap A., Slovák J., On local flatness of manifolds with AHS-structures. The Proceedings of the 15th Winter School "Geometry and Physics" (Srní, 1995). Rend. Circ. Mat. Palermo (2) Suppl. No. 43 (1996), 95-101.

[DG] Dragovic V., Gajic B., The Wagner Curvature Tensor in Nonholonomic Mechanics, Reg. Chaot. Dyn., 2003, 8 (1), 105-124; math-ph/0304018

[EKMR] Ehlers, K.; Koiller, J.; Montgomery, R.; Rios, P. Nonholonomic systems via moving frames: Cartan equivalence and Chaplygin Hamiltonization. The breadth of symplectic and Poisson geometry, Progr. Math., 232, Birkhäuser Boston, Boston, MA, 2005, 75-120

[FF] Feigin B., Fuchs D., Cohomology of Lie groups and Lie algebras. In: Lie groups and Lie algebras - 2. Itogi nauki i theniki. Sovremennye problemy matematiki. Fundamental'nye napravleniya, v. 21, VINITI, 1988, 121-209 (= English translation by Springer in Sov. Math. Encyclop. series)

[FG] Fock V.V., Goncharov A.B., Cluster X-varieties, amalgamation and Poisson-Lie groups, math.RT/0508408

[GIOS] Galperin, A. S.; Ivanov, E. A.; Ogievetsky, V. I.; Sokatchev, E. S. Harmonic superspace. Cambridge Monographs on Mathematical Physics. Cambridge University Press, Cambridge, 2001. xiv+306 pp.

[Go] Goncharov A., Infinitesimal structures related to hermitian symmetric spaces, Funct. Anal. Appl, 15, no. 3 (1981), 23-24 (Russian); a detailed version: id., Generalized conformal structures on manifolds. Selected translations. Selecta Math. Soviet. 6 (1987), no. 4, 307-340

[Gr] Grozman P., SuperLie, http://www.equaonline.com/math/SuperLie

[GL] Grozman P., Leites D., SuperLie and problems (to be) solved with it. Preprint MPIM-Bonn, 2003-39 (http://www.mpim-bonn.mpg.de)

[GL1] Grozman P., Leites D., Supergravities and $N$-extended Minkowski superspaces for any $N$. In: Wess J., Ivanov E. (eds.) Supersymmetries and quantum symmetries. Proc. International Conference in memory of V. Ogievetsky, June 1997, Lecture Notes in Physics 524, Springer, 1999, 58-67

[GL2] Grozman P., Leites D., Defining relations for Lie superalgebras with Cartan matrix. Czechoslovak J. Phys. 51 (2001), no. 1, 1-21; arXiv: hep-th/9702073

[GLS] Grozman P., Leites D., Shchepochkina I., The analogs of the Riemann tensor for exceptional structures on supermanifolds. In: S. K. Lando, O. K. Sheinman (eds.) Proc. International conference "Fundamental Mathematics Today" (December 26-29, 2001) in honor of the 10th Anniversary of the Independent University of Moscow, IUM, MCCME 2003, 89-109; preprint MPIM-2003-18 (http://www.mpim-bonn.mpg.de) 
[H] Hertz H., The principles of mechanics in new relation. NY, Dover, 1956

[HH] Heslop, P.; Howe, P. S. On harmonic superspaces and superconformal fields in four dimensions. Classical Quantum Gravity 17 (2000), no. 18, 3743-3768.

[K] Kostant, B., A generalization of the Bott-Borel-Weil theorem and Euler number multiplets of representations Conférence Moshé Flato 1999, Vol. I (Dijon), 309-325, Math. Phys. Stud., 21, Kluwer Acad. Publ., Dordrecht, 2000.

id., ibid., Conference Moshé Flato 1999 (Dijon). Lett. Math. Phys. 52 (2000), no. 1, 61-78

[Koz] Kozlov V., The heat equilibrium in the sense of Hibbs and Poincaré. Institure for computer research, Moscow-Izhevsk, 2002, 320 pp (in Russian)

[LLS] Lebedev A., Leites D., Shereshevskii I., Lie superalgebra structures in cohomology spaces of Lie algebras with coefficients in the adjoint representation, arXiv: math.KT/0404139 In: É. Vinberg (ed.) Lie groups and invariant theory, A. L. Onishchik Festschrift, Amer. Math. Soc. Transl. Ser. 2, 213, Amer. Math. Soc., Providence, RI, 2005,157-172.

[L] Leites D., The Riemann tensor for nonholonomic manifolds. Homology, Homotopy and Applications, vol 4 (2), 2002, 397-407; math.RT/0202213 (for Lecture notes, see: Workshop on mathematical physics and geometry, ICTP, Trieste, March 4-15, 1991. Repository for lecture notes from the scanned historical archive, http://agenda.ictp.trieste.it/agenda/current/fullAgenda.php?ida=a02210)

[LPS] Leites D., Poletaeva E., Serganova V., On Einstein equations on manifolds and supermanifolds, J. Nonlinear Math. Physics, 2002, no. 4, 394-425; math.DG/0306209

[LP] Leites D., Poletaeva E., Supergravities and contact type structures on supermanifolds. Second International Conference on Algebra (Barnaul, 1991), Contemp. Math., 184, Amer. Math. Soc., Providence, RI, 1995, 267-274

[LSV] Leites D., Serganova V., Vinel G., Classical superspaces and related structures. In: Bruzzo U. e.a. (eds.) Proc. Intnl. Conf. Diff. Geom. Methods in Physics DGM-IXI, 1990, Springer LN in Phys., 1991, 286-297

[LSc] Leites D., Shchepochkina I., How to quantize the antibracket, preprint ESI-875 (http://www.esi.ac.at); Theor. and Math. Physics, v. 126, 2001, no. 3, 339-369;

[LSh] Leites D., Shchepochkina I., Classification of the simple Lie superalgebras of vector fields, preprint MPIM-2003-28 (http://www.mpim-bonn.mpg.de)

[MaG] Manin Yu. Gauge field theory and complex geometry. Springer-Verlag, Berlin, 1997. xii+346 pp.

[M] Montgomery R., Engel deformations and contact structures. Northern California Symplectic Geometry Seminar, 103-117, Amer. Math. Soc. Transl. Ser. 2, 196, Amer. Math. Soc., Providence, RI, 1999

[OV] Onishchik A., Vinberg E., Seminar on algebraic groups and Lie groups, Springer, 1987

[OT] Ovsienko V., Tabachnikov S., Projective differential geometry, old and new: from Schwarzian derivative to cohomology of diffeomorphism groups, Cambridge University Press, in print (http://www.math.psu.edu/tabachni/prints/preprints.html)

[Pe] Penkov I., Borel-Weil-Bott theory for classical Lie supergroups. Itogi Nauki i Tekhniki, Current problems in mathematics. Newest results, Vol. 32, 71-124, Akad. Nauk SSSR, Vsesoyuz. Inst. Nauchn. i Tekhn. Inform., Moscow, 1988. (Russian) Translated in J. Soviet Math. 51 (1990), no. 1, 2108-2140.

[PS] Penkov I., Serganova V., Cohomology of $G / P$ for classical complex Lie supergroups $G$ and characters of some atypical $G$-modules. Ann. Inst. Fourier (Grenoble) 39 (1989), no. 4, 845-873.

[Poi] Poincaré H., Ideas of H. Hertz in mechanics. In: Избранные труды в трех томах. (Russian) [Selected works in three volumes] Том III: Математика. Теоретическая физика. Анализ математических и естественнонаучных работ Анри Пуанкаре. [Volume III: Mathematics. Theoretical physics.], Nauka, Moscow, 1974. (Les idées de Hertz en mécanique. (French) Rev. gen. des sc. 8, 1897, 734-743.)

[P] Poletaeva E., Analogs of the Riemannian tensor on supermanifolds, MPIM-2003-19, 71 pp (http://www.mpim-bonn.mpg.de)

[S] Sergeev V., The limits of rationality. (A thermodynamic approach to market equilibrium), Fasis, Moscow, 1999, v+144 (in Russian); for a draft of translation with commentaries, see [S1]

[S1] Sergeev V., The thermodynamical approach to market economy). Translated into English and edited by D. Leites. With Appendices by D. Leites, G. Skorobogatov and A. Konyaeva, A. Vershik. ca 200 pp. ???

[Sh1] Shchepochkina I. Exceptional simple infinite dimensional Lie superalgebras, C.R. Acad. Sci. bulg. $1983,36, \mathrm{n} 3,313-314$ 
[Sh2] Shchepochkina I., The five exceptional simple Lie superalgebras of vector fields, hep-th 9702120 Five simple exceptional Lie superalgebras of vector fields. (Russian) Funktsional. Anal. i Prilozhen. 33 (1999), no. 3, 59-72, 96; translation in Funct. Anal. Appl. 33 (1999), no. 3, 208-219 (2000)

[Sh14] Shchepochkina I., Five exceptional simple Lie superalgebras of vector fields and their fourteen regradings. Representation Theory (electronic journal of AMS), v. 3, 1999, 3 (1999), 373-415

[ShN] Shchepochkina I., How to realize Lie algebras by vector fields., ???

[St] Sternberg S. Lectures on differential geometry, 2nd ed, Chelsey, 1985

[T] Tanaka N., On infinitesimal automorphisms of Siegel domains, J. Math. Soc. Japan 22, 1970, 180-212; id., On the equivalence problems associated with simple graded Lie algebras. Hokkaido Math. J., 8 (1979), no. 1, 23-84

[Ta] Tavares, J. N. About Cartan geometrization of non-holonomic mechanics. J. Geom. Phys. 45 (2003), no. $1-2,1-23$

[Va6] Vacaru S., Interactions, strings and isotopies in higher order anisotropic superspaces. Hadronic Press Monographs in Mathematics. Hadronic Press, Inc., Palm Harbor, FL, 1998. ii+449 pp.

[V] Vershik, A. M. Classical and nonclassical dynamics with constraints. (Russian) In: Yu. G. Borisovich and Yu. E. Gliklikh (eds.) Geometry and topology in global nonlinear problems, 23-48, Novoe Global. Anal., Voronezh. Gos. Univ., Voronezh, 1984. Very poor English translation in: Yu. G. Borisovich and Yu. E. Gliklikh (eds.), Global analysis-studies and applications. I. A collection of articles translated from the Russian. Lecture Notes in Mathematics, 1108. Springer-Verlag, Berlin-New York, 1984, 278301 ; for an edited translation, see [S1].

[VG2] Vershik A., Gershkovich V. Nonholonomic dinamical systems. In: Fundamental trends, VINITI, Moscow, 1987, 5-85 (translated by Springer in Sov. Math. Encyclop. series "Dynamical systems-7")

[VG] Gershkovich, V.; Vershik, A. Nonholonomic manifolds and nilpotent analysis. J. Geom. Phys. 5 (1988), no. 3, 407-452;

Vershik, A. M.; Gershkovich, V. Ya. A bundle of nilpotent Lie algebras over a nonholonomic manifold (nilpotentization). (Russian) Zap. Nauchn. Sem. Leningrad. Otdel. Mat. Inst. Steklov. (LOMI) 172 (1989), Differentsialnaya Geom. Gruppy Li i Mekh. Vol. 10, 21-40, 169 translation in J. Soviet Math. 59 (1992), no. 5, 1040-1053

[WB] Wess J., Bagger J., Supersymmetry and supergravity. Second edition. Princeton Series in Physics. Princeton University Press, Princeton, NJ, 1992. x+259 pp.

[Wo] Woit P., Quantum Field Theory and Representation Theory: A Sketch. arXiv: hep-th/0206135

[Y] Yamaguchi K., Differential systems associated with simple graded Lie algebras. Progress in differential geometry, Adv. Stud. Pure Math., 22, Math. Soc. Japan, Tokyo, 1993, 413-494

[YY] Yamaguchi, K.; Yatsui, T. Geometry of higher order differential equations of finite type associated with symmetric spaces. In: Morimoto T, Sato H., and Yamaguchi K. (eds.) Proceedings of the International Conference held at Kyoto University, Kyoto, and at Nara Women's University, Nara, December 1999. Advanced Studies in Pure Mathematics, 37. Mathematical Society of Japan, Tokyo, Lie groups, geometric structures and differential equations - one hundred years after Sophus Lie (Kyoto/Nara, 1999), Math. Soc. Japan, Tokyo, 2002, 397-458

[Z] Zhitomirskii, M., Typical singularities of differential 1-forms and Pfaffian equations. Translated from the Russian. Translations of Mathematical Monographs, 113. American Mathematical Society, Providence, RI; in cooperation with Mir Publishers, Moscow, 1992. xii+176 pp.

${ }^{1}$ Equa Simulation AB, Stockholm, Sweden; Pavel@rixetele.com, ${ }^{2}$ MPimis, Inselstr. 22, DE-04103 Leipzig, Germany, on leave from Department of Mathematics, University of Stockholm, Roslagsv. 101, Kräftriket hus 6, SE-10405 Stockholm, Sweden, mleites@math.su.se 